\title{
Hybrid-COVID: A novel hybrid 2D/3D CNN based on cross-domain adaptation approach for COVID-19 screening from chest $X$-ray images
}

\section{Khaled Bayoudh ( $\sim$ Khaled.isimm@gmail.com )}

Electrical Department, National Engineering School of Monastir (ENIM), Laboratory of Electronics and Micro-electronics (LR99ES30), Faculty of Sciences of Monastir (FSM), University of Monastir, Monastir, Tunisia https://orcid.org/0000-0002-1148-4800

\section{Fayçal Hamdaoui}

Electrical Department, National Engineering School of Monastir (ENIM), Laboratory of Control, Electrical Systems and Environment (LASEE), National Engineering School of Monastir, University of Monastir, Monastir, Tunisia

\section{Abdellatif Mtibaa}

Electrical Department, National Engineering School of Monastir (ENIM), Laboratory of Electronics and Micro-electronics (LR99ES30), Faculty of Sciences of Monastir (FSM), University of Monastir, Monastir, Tunisia

\section{Research Article}

Keywords: COVID-19, Chest X-ray, Hybrid 2D/3D CNN, Deep learning, Pneumonia

Posted Date: October 9th, 2020

DOl: https://doi.org/10.21203/rs.3.rs-89931/v1

License: (c) (1) This work is licensed under a Creative Commons Attribution 4.0 International License. Read Full License

Version of Record: A version of this preprint was published at Physical and Engineering Sciences in Medicine on December 1st, 2020. See the published version at https://doi.org/10.1007/s13246-02000957-1. 


\title{
Hybrid-COVID: A novel hybrid 2D/3D CNN based on cross-domain adaptation approach for COVID-19 screening from chest X-ray images
}

\author{
Khaled Bayoudh • Fayçal Hamdaoui . \\ Abdellatif Mtibaa
}

Received: date / Accepted: date

\begin{abstract}
So far, COVID-19, the novel coronavirus, continues to spread rapidly in most countries of the world, putting people's lives at risk. According to the WHO, respiratory infections occur primarily in the majority of patients treated with COVID-19. For decades, chest X-ray (CXR) technologies have proven their ability to accurately detect and treat respiratory diseases. Deep learning techniques, as well as the availability of a large number of CXR samples, have made a significant contribution to the fight against this pandemic. However, the most common screening methods are based on 2D CNNs, since $3 \mathrm{D}$ counterparts are enormously costly and labor-intensive. In this study, a hybrid 2D/3D convolutional neural network (CNN) architecture for COVID-19 screening using CXRs has been developed. The proposed architecture consists of the incorporation of a pre-trained deep model (VGG-16) and a shallow 3D $\mathrm{CNN}$, combined with a depth-wise separable convolution layer and a spatial pyramid pooling module (SPP). Specifically, the depth-wise separable convolution helps to preserve the useful features while reducing the computational burden of the model. The SPP module is designed to extract multi-level representations from intermediate ones. Experimental results show that the pro-

K. Bayoudh

Electrical Department, National Engineering School of Monastir (ENIM), Laboratory of Electronics and Micro-electronics (LR99ES30), Faculty of Sciences of Monastir (FSM), University of Monastir, Monastir, Tunisia

E-mail: khaled.isimm@gmail.com

ORCID: 0000-0002-1148-4800

F. Hamdaoui

Electrical Department, National Engineering School of Monastir (ENIM), Laboratory of Control, Electrical Systems and Environment (LASEE), National Engineering School of Monastir, University of Monastir, Monastir, Tunisia

A. Mtibaa

Electrical Department, National Engineering School of Monastir (ENIM), Laboratory of Electronics and Micro-electronics (LR99ES30), National Engineering School of Monastir, University of Monastir, Monastir, Tunisia
\end{abstract}


posed framework can achieve reasonable performances when evaluated on a collected dataset (3 classes: COVID-19, Pneumonia, and Normal). Notably, it achieved a sensitivity of $98.33 \%$, a specificity of $98.68 \%$ and an overall accuracy of $96.91 \%$

Keywords COVID-19 · Chest X-ray · Hybrid 2D/3D CNN · Deep learning · Pneumonia

\section{Introduction}

The novel coronavirus (COVID-19), which originated in Wuhan, China at the end of 2019, has become a serious threat worldwide to public health [1. Accordingly, this pandemic stands as a global health emergency. The continuing spread of the COVID-19 pandemic, which claims a large number of victims and serious infections every day, affecting several territories, such as the United States, Italy, Spain, etc., makes its treatment increasingly challenging. According to the world health organization (WHO), COVID-19 has a considerable impact on human beings. The majority of infected patients suffer from mild to moderate respiratory issues, the severity of which changes over time [2]. According to [3], COVID-19 disease may occur asymptomatically in up to $20 \%$ of infected patients but can be transmitted rapidly to many people. However, international strategies to fight this epidemic differ from one country to another depending on the strength of the health infrastructure and the level of public awareness. For example, in Tunisia, the disease has spread slowly compared to Europe and Asia, thanks to early efforts and measures taken by the authorities to reduce its rapid transmission in the country [25]. Currently, the most recognized strategy to fight the pandemic involves intensified screening for the infection. To slow down its spread, millions of people still need to be screened over time. The real-time reverse transcriptase-polymerase chain reaction (RTPCR) test can detect and screen the presence of the virus with a high-level of sensitivity. This kind of test has often been used as the main screening process for COVID-19 by directly identifying the existence of the virus. Despite this advantage, RT-PCR suffers from some drawbacks, such as its complexity and certain specificity issues. Moreover, it is very time-consuming and expensive especially when the collected specimens need to be processed by external specialized laboratories. Hence, this makes it very difficult to perform RT-PCR testing for a large number of suspected patients in as short a time as possible. To address these shortcomings, researchers have developed an important complement to RT-PCR tests by detecting COVID-19 from chest computed tomography (CT) scans [4] 7]. This will help reduce treatment delays and patient isolation. In 4], the authors concluded that chest CT scans were more sensitive for the diagnosis of COVID-19 than traditional RT-PCR. In [5], the authors showed that the chest CT scanner has considerable performance in diagnosing COVID-19 in terms of speed, specificity, and sensitivity. A comparative study of 51 patients showed that chest CT scans were very sensitive (50/51 patients) for screening for COVID-19 disease [6]. In a study by [7], a 
total of 219 COVID-19 positive patients detected by both RT-PCR and chest $\mathrm{CT}$ were conducted. The researchers concluded quantitatively that the distinction of COVID-19 from other types of pneumonia on CT chest scans was more sensitive than RT-PCR tests. Regardless of these strengths, CT scans still have some limitations. Among these is the fact that screening for COVID-19 generally takes much longer to perform. Moreover, high-quality scanners are very expensive and patients have to deal with much higher radiation doses 11 . In contrast, chest X-rays (CXRs) are one of the most common large-scale medical imaging techniques that have proven to be effective in speeding-up and facilitating the screening of the pandemic 12, 47]. These techniques allow the early detection and tracking of the virus. Fig. 1 shows CXRs of an older man patient from Wuhan, China [31. These samples indicate the progression of lung consolidation from day 0 , day 4 , and day 7 , respectively. However, COVID-19 patients have some clinical features and symptoms that make it distinctive from other types of viral pneumonia 8,24. For example, the authors in 8] compared the difference between COVID-19 pneumonia and other cases of pneumonia. They found that the patients' features were very similar and the most common symptoms were fever and cough. Generally, the early symptoms that make COVID-19 infection different from other viruses were fever, dry cough, heavy sweating, and weakness. Consequently, it was difficult to distinguish COVID-19 pneumonia when compared to other cases of pneumonia. AI-based methods have been widely used in many real-world applications, including healthcare, medical imaging, and so on. For example, AI-based CT scans were introduced to distinguish COVID-19 features from other diseases 9. Currently, the development of new diagnostic methods using deep learning algorithms is underway. Convolutional Neural Networks (CNNs) have been one of the deep neural networks (DNNs) used to screen for COVID-19 from patient data, leading to impressive results [10]. Nowadays, the availability of large-scale CT and CXR image datasets for deep model training and testing purposes has encouraged the deep learning community to develop intelligent systems for the screening of coronavirus disease $10,13,14$. For instance, the results obtained in 10 showed that the use of large CT datasets $(618$ samples in total) for the training model led to reliable COVID-19 diagnostic outcomes. Since the beginning of the pandemic, many researchers have proposed a range of deep learning-based methods for COVID-19 detection and diagnosis from X-ray scans, including $18,22,36,37$. In [18, a deep model based on CXR data showed a high accuracy of up to $98 \%$. The network parameters were loaded from a pre-trained ResNet50 24] model. Similarly, the authors of 19 used the transfer learning technique with CNNs to speed-up the detection of COVID-19 on X-ray samples. They achieved an accuracy, sensitivity, and specificity of $96.78 \%, 98.66 \%$, and $96.46 \%$, respectively. In [20], the authors proposed a screening model based on ensemble learning techniques, called "DeepCOVIDExplainer", which was used to predict the COVID-19 pandemic from X-ray features (16,995 samples in total). The authors of [21] introduced a binary classification model (i.e. 2 classes to be predicted: COVID-19 or non-COVID-19, respectively) using ResNet-18 as the backbone network. The 
backbone network was used to extract visual patterns from CXR images following the transfer learning paradigm. They reached a sensitivity of $96.00 \%$ and a specificity of $70.65 \%$ on 1531 X-ray images. In [22], the authors developed a powerful deep learning framework, called COVIDX-Net, based on CXR images and deep CNN models (e.g. VGG, ResNet, etc). The best-proposed model received F1-scores of 89\% (normal cases) and 91\% (COVID-19 cases), respectively. Ozturk T. et al 36 introduced a deep learning-based algorithm for booth binary and multi-class classification to discriminate COVID-19 patients from other diseases in CXR images. The model was produced with an accuracy of $98.08 \%-87.02 \%$ for binary and multi-class scenarios. In 37], the authors developed a novel screening system based on Fuzzy Color and Social Mimic optimization techniques for automated classification of CXR images. The overall classification rate of $99.27 \%$ was the outcome of the model. The authors of 46 proposed the Truncated Inception Net to detect COVID-19 positive cases from other non-COVID cases. It should be noted that all these methods were based on 2D CNN architectures. For decades, most imagingbased screening solutions have been based on 2D CNNs, as 3D CNN models require a lot of hardware and memory resources. In other words, end-to-end learning of a deep 3D CNN architecture may lead to a tremendous expansion of feature dimensionality and parameter space after stacking several layers of 3D convolutions. Nevertheless, an adaptive combination of a pre-trained deep 2D CNN with a 3D CNN allows better leveraging of the dynamic synergy between the multi-dimensional activation maps 32. For instance, the authors of 32 have combined 2D-3D CNNs based on the transfer learning paradigm to ensure a better compromise between accuracy and computational burden. In this paper, a faster and more accurate deep learning system has been developed for screening the COVID-19 pandemic based on the combination of a deep 2D CNN pre-trained on ImageNet (i.e. VGG16 15]) with a shallow 3D CNN, a depth-wise separable convolution [16], and a spatial pyramid pooling module 17, using CXR samples. More specifically, our idea is to transfer the $2 \mathrm{D}$ parameters learned from a $2 \mathrm{D} \mathrm{CNN}$ and integrate them into a shallow $3 \mathrm{D}$ CNN, so that extra contextual information gets provided and the training process is faster. The main innovation presented in this paper is the introduction of a novel hybrid 2D/3D CNN-based framework to efficiently and effectively diagnose COVID-19 from CXR images. To our knowledge, we are the first to propose a hybrid model for the screening of COVID-19 using CXR images.

\section{Methodology}

\subsection{Dataset}

As part of this work, we collected a total of 3440 CXR samples (without data augmentation) for the training and testing of our model. Indeed, these samples are extracted and combined from three publicly available CXR datasets 13 , 14, 35], in which COVID-19 patients, Pneumonia patients, and Normal cases 


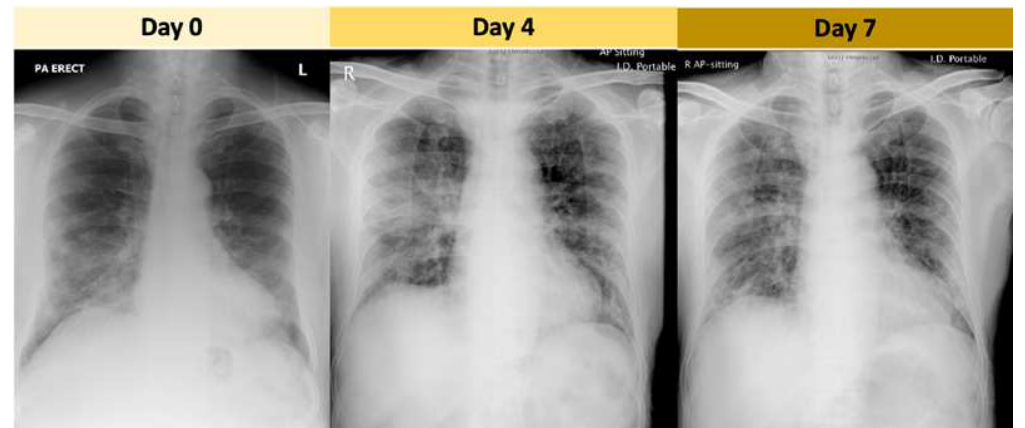

Fig. 1 Temporal evolution of CXR outcomes in an older male patient with 3 consolidation changes in the lung periphery 31 .

are labeled for final prediction. More specifically, 754 COVID-19 confirmed cases, 1341 Normal cases, and 1345 Pneumonia cases are extracted from both the COVID-19 datasets 13, 35], and the CXR (Pneumonia) dataset [14, respectively. Pneumonia patient dataset consists of information on viral and bacterial infections from a total of 53 selected cases. The datasets mentioned are regularly updated with additional data items provided by external contributors (e.g., researchers, radiologists, etc.). To reduce the imbalance in data distribution and improve generalizability, the conventional data augmentation technique is applied as a pre-processing step by rotating each CXR image by up to $20^{\circ}$. Table 1 shows the statistical distribution of data obtained before and after increasing the number of data samples. We randomly divided the augmented dataset into two distinct sets; one set of $80 \%$ for training and validation and one set of $20 \%$ for testing. The validation set is used to select the best hyper-parameters when the model performs best. Fig. 7 illustrates an example of CXR images from Normal cases (Fig. 2 (a)), COVID-19 patients (Fig. 2(b)), and Pneumonia cases (Fig. 2 (c)).

Table 1 Number of CXR images before and after the increase in data distribution for each class

\begin{tabular}{llll}
\hline Data augmentation & Class & Number of images (Train+Test) & Total \\
\hline Before & COVID-19 & 754 & \\
& Normal & 1341 & 3440 \\
& Pneumonia & 1345 & \\
\hline \multirow{2}{*}{ After } & COVID-19 & 1268 & 6370 \\
& Normal & 2547 & \\
\hline
\end{tabular}




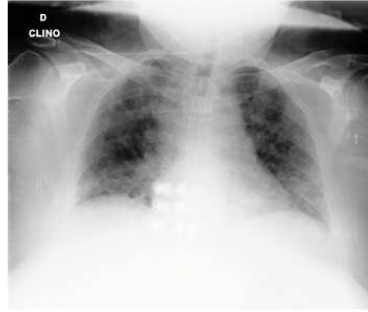

(a)

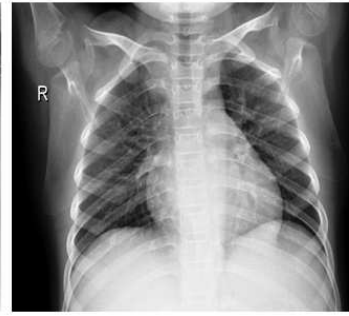

(b)

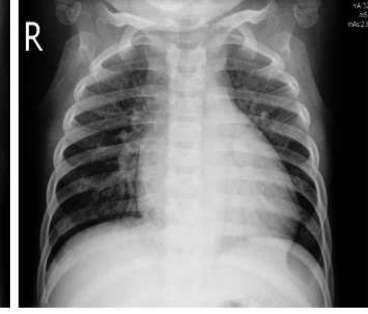

(c)

Fig. 2 A selection of CXR samples taken from the collected dataset: a Normal case (a), a COVID-19 patient (b), a Pneumonia case (c)

\subsection{Proposed method}

This section describes the proposed transfer learning-based method for end-toend screening of COVID-19 infected patients from CXR images. In practice, transfer learning, commonly known as cross-domain adaptation [33, aims to strengthen the generalization capacity of the target model by loading knowledge from the source one. Moreover, this paradigm can effectively contribute to overcoming the lack of available CXR datasets. So, in light of the specular success of transfer learning techniques as well as the generalizability of 2D and 3D CNN, we propose a hybrid framework, called Hybrid-COVID, for COVID-19 prediction. Therefore, we chosed the VGG16 pre-trained model as a baseline network for categorizing the CXR images into COVID-19, Normal, and Pneumonia. Fig. 3 show the workflow diagram of the proposed framework, which is composed mainly of six blocks, namely, respectively, a pre-trained 2D CNN (VGG16 network), an up-sampling step, a depth-wise separable convolution, a shallow 3D CNN (building block), a spatial pyramid pooling (SPP) module, followed by the classification scheme (dense layers). Inspired by the work of [32], the VGG16 model is used as a backbone network for extracting generic features from a huge dataset (i.e. ImageNet). This pre-trained model has already achieved high performance for the image classification task. The up-sampling step plays a crucial role, as it is useful to reconstruct the loaded feature maps without skipping any spatial information. Besides, the depth-wise separable convolution ensure that useful features are preserved while reducing the model's computational cost. The computational complexity of stacking multiple layers of 3D convolution layers could be overcome by fine-tuning a pre-trained model, making it easier to learn deeper and more informative representations. This involves significantly reducing network parameters as well as retaining $3 \mathrm{D}$ spatial information that is otherwise missed by the $2 \mathrm{D} \mathrm{CNN}$. A systematic integration of $2 \mathrm{D}$ and $3 \mathrm{D}$ convolutions can be achieved by reshaping their output activations through merging operations. An SPP module is then suitable for extracting multi-scale representations from intermediate levels. In the following subsections, we will describe in detail each block of the proposed Hybrid-COVID model. 
Table 2 Output shapes of the proposed architecture

\begin{tabular}{|c|c|}
\hline Layer & Output shape \\
\hline Input & $112 \times 112 \times 3$ \\
\hline Conv2D & $112 \times 112 \times 64$ \\
\hline Conv2D & $112 \times 112 \times 64$ \\
\hline MaxPooling2D & $56 \times 56 \times 64$ \\
\hline Conv2D & $56 \times 56 \times 128$ \\
\hline Conv2D & $56 \times 56 \times 128$ \\
\hline MaxPooling2D & $28 \times 28 \times 128$ \\
\hline Conv2D & $28 \times 28 \times 256$ \\
\hline Conv2D & $28 \times 28 \times 256$ \\
\hline Conv2D & $28 \times 28 \times 256$ \\
\hline MaxPooling2D & $14 \times 14 \times 256$ \\
\hline Conv2D & $14 \times 14 \times 512$ \\
\hline Conv2D & $14 \times 14 \times 512$ \\
\hline Conv2D & $14 \times 14 \times 512$ \\
\hline MaxPooling2D & $7 \times 7 \times 512$ \\
\hline Conv2D & $7 \times 7 \times 512$ \\
\hline Conv2D & $7 \times 7 \times 512$ \\
\hline Conv2D & $7 \times 7 \times 512$ \\
\hline MaxPooling2D & $3 \times 3 \times 512$ \\
\hline UpSampling2D & $6 \times 6 \times 512$ \\
\hline Depth-wiseConv2D & $3 \times 3 \times 512$ \\
\hline Reshape & $3 \times 3 \times 512 \times 1$ \\
\hline Conv3D & $3 \times 3 \times 512 \times 5$ \\
\hline Conv3D & $3 \times 3 \times 512 \times 5$ \\
\hline Conv3D & $3 \times 3 \times 512 \times 5$ \\
\hline Reshape & $3 \times 3 \times 2560$ \\
\hline Spatial Pyramid Pooling & 35840 \\
\hline Dense & 64 \\
\hline Dropout & 64 \\
\hline Dense & 3 (Output) \\
\hline
\end{tabular}

\subsubsection{Hybrid-COVID}

Hybrid-COVID is a novel transfer learning-based model architecture that aims to ensure a better compromise between accuracy and computational complexity while integrating both $2 \mathrm{D}$ and $3 \mathrm{D}$ CNNs. Table 2 shows the output shape for each level of our architecture. In the following paragraphs, each component of the model will be described in more detail.

2D CNN In computer vision, 2D CNN models have been one of the most popular deep models for image classification and understanding tasks 26]. They allow the dynamic extraction of discriminating features directly from raw data (e.g. still images, etc.) or from an intermediate level of the network. Visual Geometry Group Network (VGG) 15 is one of the deep CNN architectures that have proven their performance in extracting generic features from large datasets. To reduce the network parameters and the computational complexity, the VGG network was used a cascaded convolution layers with $3 \times 3$ filter sizes and 1 strides. This pre-trained network can be taken as a 


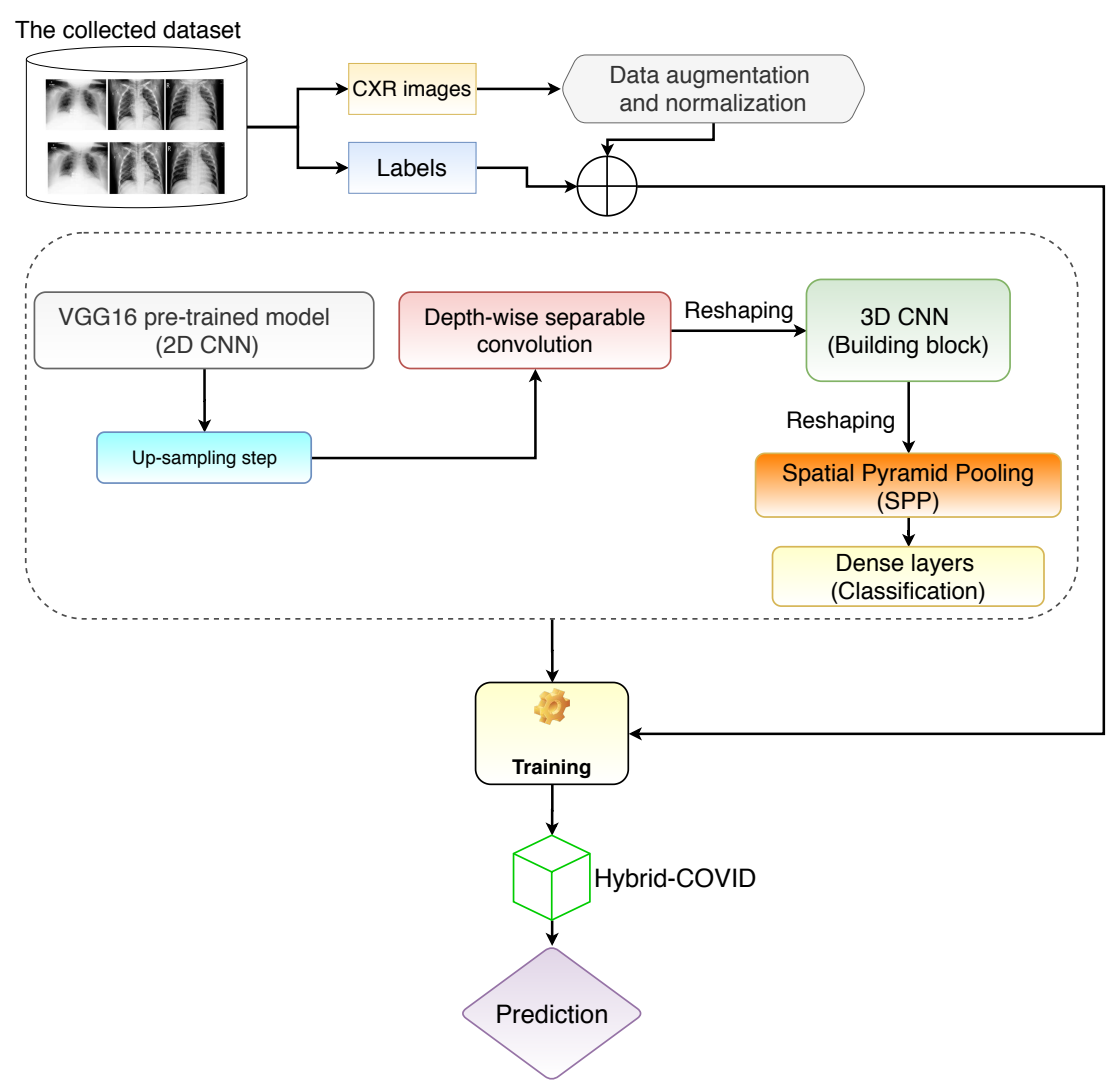

Fig. 3 Workflow diagram of the proposed framework

feature extractor by exploiting only its convolutional side. To date, this network is available in two versions, VGG16 and VGG19, which differ in their depth and hyper-parameters. In this work, we used VGG16 since it was lighter and cheaper than VGG19 for model training. Originally, the VGG16 network consists of several hidden layers (i.e. the convolutional side), including 13 convolution layers, and 3 visible layers (i.e. dense layers).

Up-sampling step Inspired by $[38$ and in order to increase the spatial dimension of the internal representations, an up-sampling operation is systematically used after loading the VGG16 pre-trained model parameters. Technically, this operator is the inverse transformation of the down-sampling (pooling) layer. In this work, we used only one UpSampling2D layer with 2 scale factors and bicubic interpolation to increase the resolution of the pooled feature maps. In practice, this step can contribute to reducing the number of unnecessary calculations without wasting any semantic information.

Depth-wise separable convolution Our entire architecture is based on 


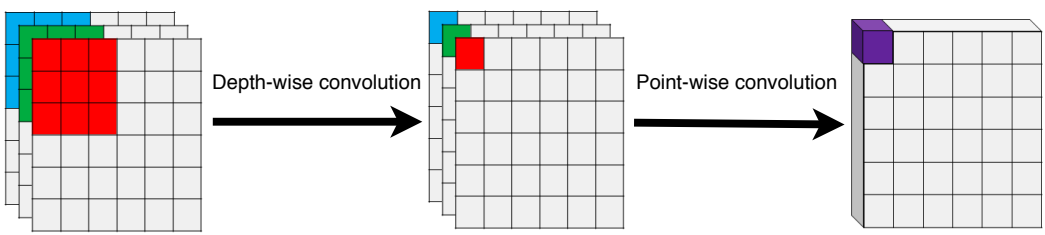

Fig. 4 Depth-wise separable convolution steps

a depth-wise separable convolution operation 16 . Indeed, it is a variant form of the regular convolution operation. In regular convolution, the filter window is applied to multiple input channels, allowing the channels to be mixed separately to produce an output feature map. In contrast, the depth-wise separable convolution operation convolves each input channel separately, after which a point-wise convolution will be performed to combine the resulting output channels. In other words, a $2 \mathrm{D}$ convolution with a filter size of $3 \times 3$ will be factorized into a depth-wise convolution of $3 \times 3$ and a point-wise convolution of $1 \times 1$, while capturing spatial and cross-channel correlations. Formally, let consider a standard convolutional kernel $\mathrm{K}$ of size $\mathrm{W} \times \mathrm{W} \times \mathrm{M} \times \mathrm{N}$. After its application on the input representation I, the output feature map $\mathrm{F}$ will be

$$
F_{k, l, n}=\sum_{i, j, m} K_{i, j, m, n} \cdot I_{k+i, l+j-1, m}
$$

where the size of the input and output representation is $\mathrm{S} \times \mathrm{S} \times \mathrm{M}$ and $\mathrm{S} \times \mathrm{S} \times \mathrm{N}$, respectively. In depth-wise separable convolution, we first extract a spatial representation from depth-wise convolution $(\tilde{K})$ of size $3 \times 3$,

$$
\tilde{I}_{k, l, n}=\sum_{i, j} \tilde{K}_{i, j, m} \cdot I_{k+i-1, l+j-1, m}
$$

we then apply the point-wise convolution $(\hat{K})$ of size $1 \times 1$ across the channel to mix the output of the depth-wise convolution. Thus, the output of the depth-wise separable convolution will be

$$
F_{k, l, n}=\sum_{m} \hat{K}_{m, n} . \tilde{I}_{k-1, l-1, m}
$$

Fig. 4 displays the steps of the depth-wise separable convolution operation. In this study, we used only a single layer with a kernel size of $3 \times 3$. The use of this operation makes it possible to reduce the network parameters and speedup the convergence rate of the model.

Fig. 5 shows some of its feature maps. As can be seen, some strong patterns (edges, textures, etc.) can be detected at this level of the network.

3D CNN Structurally, the proposed 3D CNN consists of stacking three convolution layers (i.e., a shallow network) applied with kernels of different sizes $((3 \times 3 \times 3),(2 \times 2 \times 2)$, and $(1 \times 1 \times 1)$, respectively). Note that the padding 


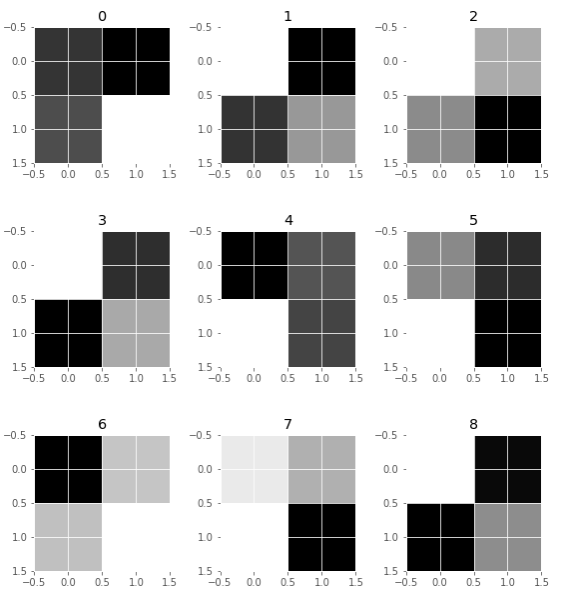

Fig. 5 Visualization of some learned weights from the depth-wise separable convolution layer

and stride hyper-parameters remain at 1 for simplicity. Also, in order to reduce the number of trainable parameters and save resources, we only set the number of filters in each layer to 5 . Compared to 2D CNN, 3D CNN shows better modeling capacity of contextual information by performing $3 \mathrm{D}$ convolutions on the space-time axes without loss of relevant features 39]. Technically, a $3 \mathrm{D}$ convolution can represent the multi-dimensional input information as a $4 \mathrm{D}$ tensor of the shape $[\mathrm{D} \times \mathrm{H} \times \mathrm{W} \times \mathrm{C}]$, where $\mathrm{D}, \mathrm{H}, \mathrm{W}, \mathrm{C}$ refers to the depth (i.e. the temporal dimension), height, width and number of slices, respectively. In this work, we used the reshaping operation to ensure the space matching of the input and output shapes while keeping the sliced data unchanged. Unlike an ordinary 3D CNN model that takes multiple input video images [34], the proposed 3D building block fine-tunes the spatial representations of the previous stage for improved sharing of knowledge and computation across domains. Formally, a set of 3D convolution kernels will be applied to the reshaped feature maps (i.e. 3D data cubes) to capture any inherited contextual patterns in the data stream. By sliding a 3D convolution kernel across the spatial and temporal dimensions, the output representation at the position (h, w, d) on the jth feature tensor of the ith layer can be computed as Eq4

$$
F_{i, j}^{h, w, d}=\Phi\left(b_{i, j}+\sum_{n} \sum_{l=0}^{L_{i}-1} \sum_{k=0}^{K_{i}-1} \sum_{t=0}^{T_{i}-1} w_{i, j, n}^{l, k, t} f_{(i-1) n}^{h+l, w+k, d+t}\right)
$$

where $\Phi$ is the activation function, also called non-linearity function, $w_{i, j, n}^{l, k, t}$ and $b_{i, j}$ are the network parameters, $\mathrm{n}$ is the index of all feature maps. $L_{i}$ and $K_{i}$ designate respectively the spatial dimensions of the $3 \mathrm{D}$ convolution kernel, while $T_{i}$ designates the temporal axis. Fig. 6 depicts a $3 \mathrm{D}$ convolution layer with $\mathrm{h} \times \mathrm{w} \times \mathrm{d}$ inputs and $\mathrm{l} \times \mathrm{k} \times \mathrm{t}$ kernel. 


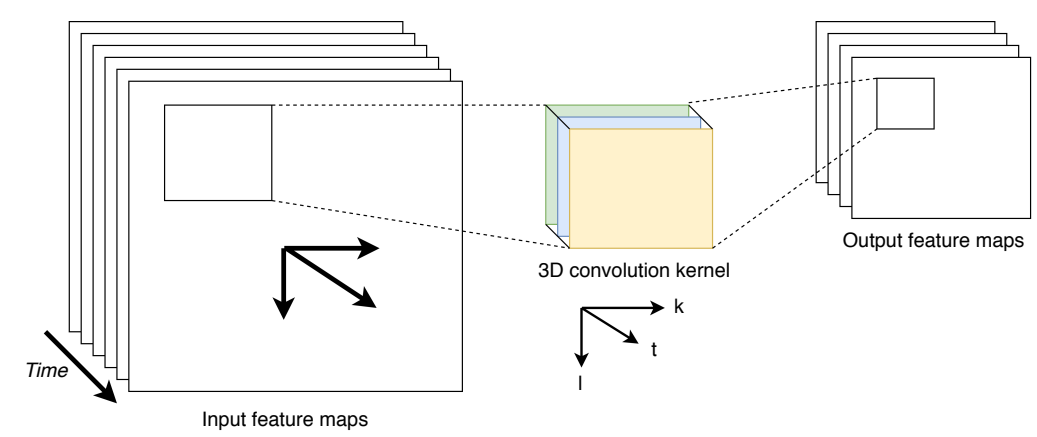

Fig. 6 A schematic illustration of a 3D convolution layer

Spatial Pyramid Pooling In this paper, we integrated the spatial pyramid pooling (SPP) module [17] in our model architecture to extract multi-scale feature maps from spatial dimensions. Technically, SPP is the dynamic variant of the pooling operation, which consists of extracting a fixed-length feature map from representations of arbitrary size by concatenating their spatial outputs. In particular, the SPP technique is used in this work to produce feature vectors of fixed dimensions for X-ray features of varying scales. This can reduce scale-variance and avoid the risk of over-fitting concern. The proposed structure of the SPP block is shown in Fig. 77. The input of the SPP block is a set of reshaped feature maps of the last 3D convolution layer. The final classification is carried out by dense layers applied on SPP-features.

\subsubsection{Hybridization process}

The goal of the hybridization process is to learn multi-domain features from multi-dimensional data cube using both 2D and 3D CNNs. The proposed Hybrid-COVID model mainly consists of two correlated sides: the 2D CNN side and the 3D CNN side. After removal of the last dense layer, the 2D CNN side (i.e., a pre-trained VGG16 network) is used to load discriminating and abstract representations that are learned from a huge dataset (i.e., 1000KImageNet). Furthermore, the 3D CNN side is fine-tuned by the $2 \mathrm{D} \mathrm{CNN}$ one to learn high-level contextual features from the reshaped data. Thus, the HybridCOVID model is produced by integrating both 2D CNN and 3D CNN through reshaping operations. This operation can be formulated as follows:

$$
R_{f}=f\left(R_{2 D}^{l}, R_{3 D}^{l}\right)
$$

where $R_{2 D}^{l}$ and $R_{3 D}^{l}$ represent the learned activations from $2 \mathrm{D}$ and 3D CNN sides, respectively. $f()$ is a function that allows the input tensors to be reshaped into a fixed-size tensor with the same rank values. 


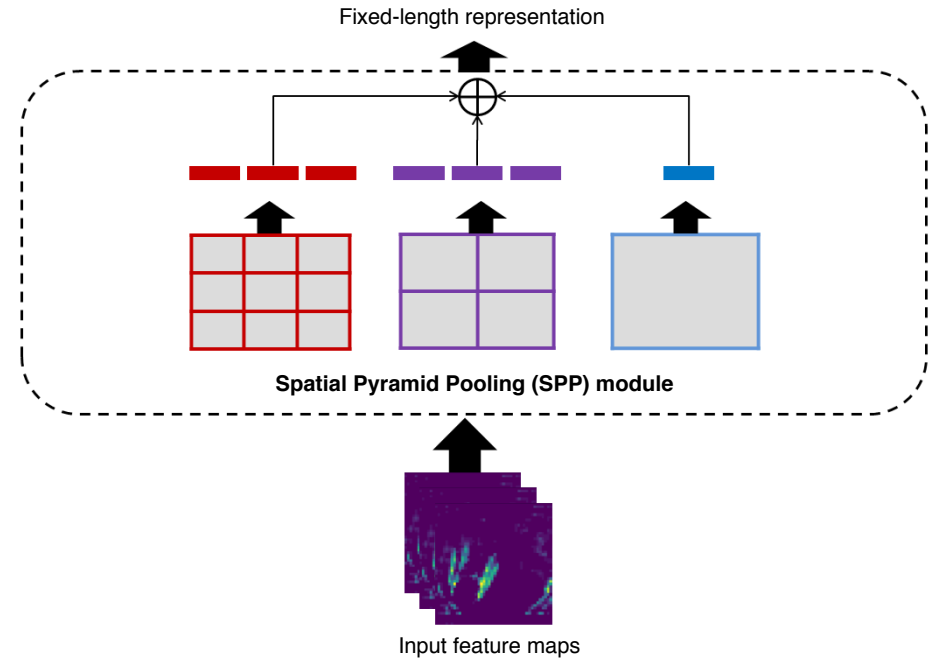

Fig. 7 Proposed spatial pyramid pooling (SPP) structure. Here, 3-level pyramid pooling is applied: $3 \times 3,2 \times 2$, and $1 \times 1$, respectively

\subsubsection{Classification scheme}

The classification scheme is performed at the end of the SPP block to categorize the input CXR image into either a positive COVID-19 case, a positive Pneumonia, or a Normal case. For this purpose, we first added a dense layer of 64 neurons, followed by the rectified linear unit (ReLU) activation function. Indeed, ReLU is one of the fastest and most effective learning activation functions that has proven its effectiveness in avoiding the problem of vanishing gradient 27]. A last dense layer of 3 neurons, followed by the Softmax activation function (i.e. a multi-class predictor) is then included to perform the final prediction.

\subsection{Training implementation}

During the training process, the network parameters will be adjusted using the Back-propagation algorithm, which iteratively decreases the loss function until convergence. In this work, the categorical cross-entropy loss is employed as the loss function. Besides, we used the Adam optimizer [28] for a faster convergence of the algorithm. We fixed the maximum epochs at 30 , the batch size at 16 , and the initial learning rate at 0.0003 . Each training sample is resized to a spatial dimension of $112 \times 112$ pixels. The one-hot encoding scheme is then applied to convert the labeled data into numerical values. For model regularization, the dropout 29] and data augmentation 30] (see sub-section 2.1) techniques are used to avoid the overfitting problem. The details of the algorithm of the proposed Hybrid-COVID model are summarized in Algorithm 1. Note that 
$\mathrm{X}$ is a set of CXR images, while $\mathrm{Y}$ represents the corresponding set of class labels. The implementation of the proposed model is achieved by using Python 3 and the publicly available Keras library with the TensorFlow 2.0 backend on Nvidia Tesla K80 GPUs with about 12 GB of memory.

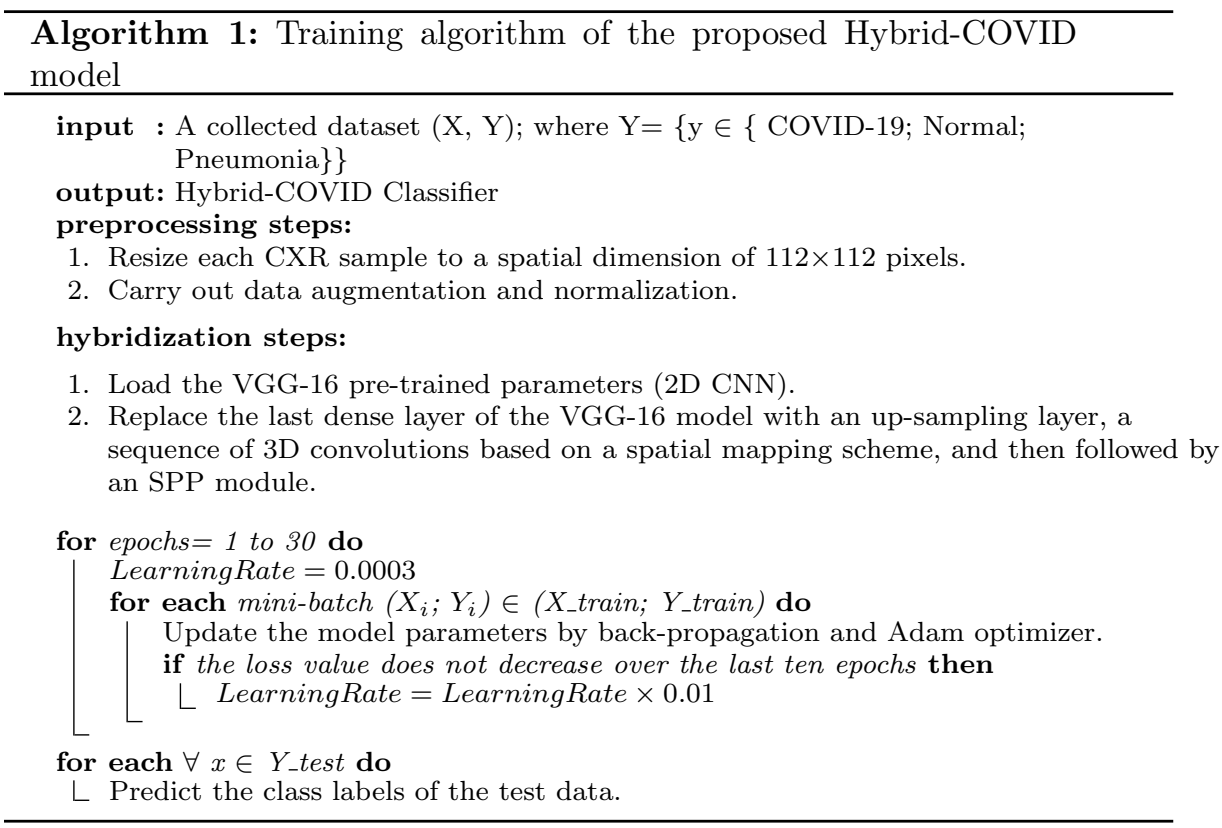

\subsection{Metrics}

The proposed model shows acceptable experimental findings in terms of accuracy and efficiency on a collected dataset. To quantitatively prove this performance, therefore, standard measures are required for empirical analysis. These metrics include Precision (PREC), F1-Score (F-SCORE), Sensitivity (SEN)/Recall (REC), Specificity (SPE), and Accuracy (ACC). The PREC measures the correct prediction rate. The F-SCORE is the harmonic mean of precision and recall, which aims to strike a balance between the two. The SEN and SPE are used to measure true positive and true negative rates, respectively. The ACC determines the percentage of correctly predicted labels of total tests. These measures can be calculated as follows:

$$
\begin{gathered}
P R E C=T P /(T P+F P) \\
F-S C O R E=2 T P /(2 T P+F P+F N) \\
S E N=T P /(T P+F N)
\end{gathered}
$$




$$
\begin{gathered}
S P E=T P /(F P+T N) \\
A C C=T P+T N /(T P+F P)+(T N+F N)
\end{gathered}
$$

Note that $T P, F P, T N$, and $F N$, respectively, stand for true positives, false positives, true negatives, and false negatives. In addition to the above measures, the confusion matrix is also included in our experiments to statistically evaluate the classification performance of the trained model by plotting the relationships between predicted values and ground-truth values. The obtained results will be analysed and discussed in the '[Results' and 'Discussion' sections of this study.

\section{Results}

The main objective of the proposed deep learning-based framework is to categorize CXR images into three categories: COVID-19, Normal, or Pneumonia. We emphasize that the proposed Hybrid-COVID is a 2D/3D hybrid architecture designed to detect patients suspected of having COVID-19 by analyzing CXR images for early screening of the disease. This section assesses the proposed model on the collected dataset and compares it with state-of-the-art methods.

\subsection{Performance evaluation}

In this study, the proposed Hybrid-COVID model is trained and evaluated on CXR samples. It seeks to ensure a better compromise between accuracy and complexity by reducing the false negative rate as much as possible (i.e. misclassification cost of diagnosed COVID-19 cases) as well as the computational time. The screening performance of the model is assessed using a set of of previously mentioned classification metrics, including confusion matrix. Nevertheless, we first assess the accuracy and loss of training and validation over epochs. These two indicators have been most often used in the literature to assess the performance of deep learning classifiers. Fig. 8 shows the evolution of training and validation accuracy and loss. As can be seen, the validation accuracy increases to a certain level of stability and reaches $95.45 \%$, while the validation loss values decrease more rapidly to $14 \%$. Moreover, the over-fitting problem has already exceeded. Fig. 9 displays the confusion matrix of our model. As we can see, the screening model predicted 250 of the COVID-19 cases as true positives, 150 and 260 of the Normal and Pneumonia classes as true negatives, respectively. Table 3 shows the class-wise classification performance of the model; we obtained a reasonable result for the COVID-19 diagnosis using our method with 99.77\% PREC, 99.67\% REC, and 99.13\% F-SCORE with a spatial resolution of $112 \times 112$. 
Table 3 Evaluation of classification performance

\begin{tabular}{llllll}
\hline Method & \multirow{2}{*}{ Resolution } & Class & PREC (\%) & REC (\%) & F-SCORE (\%) \\
\hline \multirow{2}{*}{ Hybrid-COVID } & \multirow{2}{*}{$112 \times 112$} & COVID-19 & 99.77 & 99.67 & 99.13 \\
& & Normal & 96.75 & 95.82 & 95.98 \\
& & Pneumonia & 95.83 & 96.88 & 96.24 \\
\hline
\end{tabular}

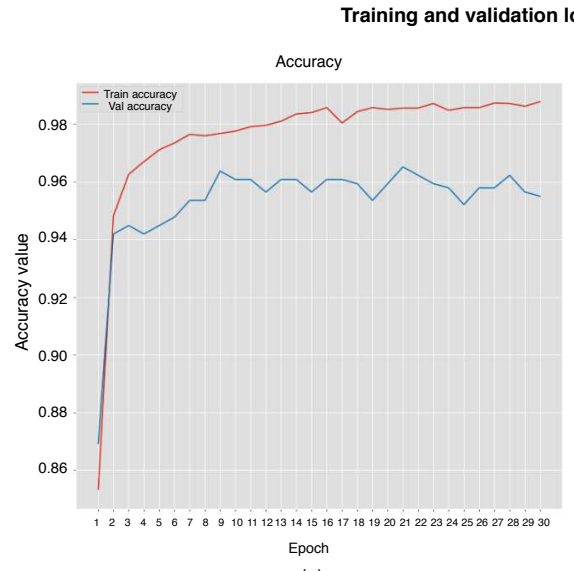

Epoch
(a)

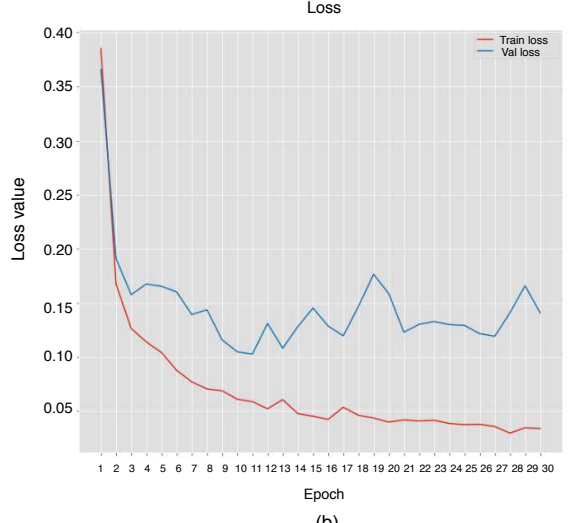

(b)

Fig. 8 Visualization of model performance per epoch for: training and validation accuracy (a), training and validation loss (b)

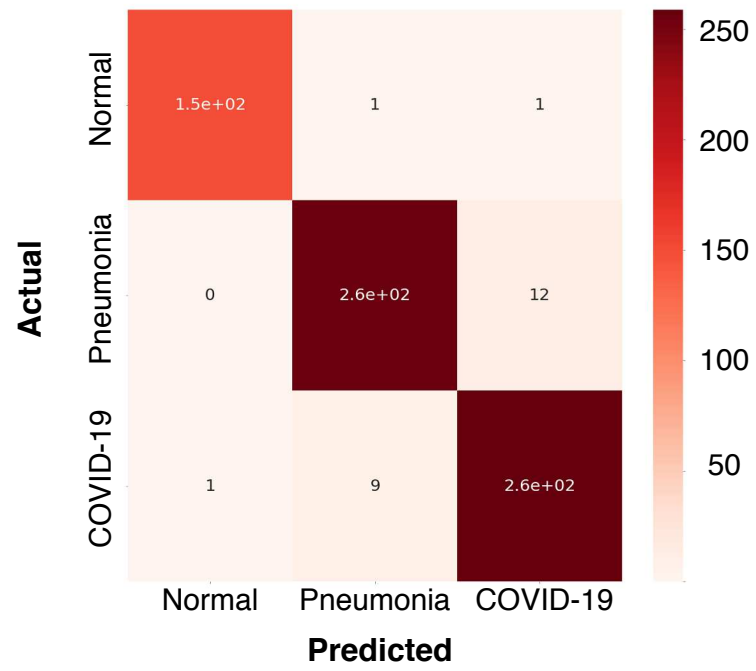

Fig. 9 Confusion matrix of the Hybrid-COVID model 
Table 4 Comparison with state-of-the art methods

\begin{tabular}{lllllll}
\hline Method & Architecture & Resolution & Number of CXR samples & SEN (\%) & SPE (\%) & ACC (\%) \\
\hline Hybrid-COVID & 2D CNN+3D CNN & $112 \times 112$ & 3440 & 98.33 & 98.68 & 96.91 \\
DarkCovidNet & 2D CNN & $256 \times 256$ & 1125 & 85.35 & 92.18 & 87.02 \\
VGG19+Transfer learning & 2D CNN & $200 \times 266$ & 1427 & 92.85 & 98.75 & 93.48 \\
CoroNet & 2D CNN & $224 \times 224$ & 1251 & 96.9 & 97.5 & 95 \\
CapsNet & 2D CNN & $128 \times 128$ & 1424 & 84.22 & 91.79 & 84.22 \\
\hline
\end{tabular}

3.2 Comparaison with state-of-the-art methods

The proposed screening system has proven its performance over state-of-theart methods:

- DarkCovidNet [36]: A framework is introduced to automatically diagnose COVID-19 infection in CXR images based on the DarkNet model [41].

- VGG19+Transfer learning 19]: A transfer learning-based framework is proposed for the early detection of COVID-19 cases. VGG19 was the best model based on experimental results.

- CoroNet [45]: An architecture based on a pre-trained Xception network is proposed to discriminate the features of COVID-19 patients from other diseases.

- CapsNet 43]: A deep learning-based architecture is introduced, allowing the detection of COVID-19 infection using CXR images and capsule networks 44.

In what follows, we will briefly outline each of these methods.

DarkCovidNet 36 achieved an ACC rate of $87.02 \%$ on a CXR dataset of 1125 images, divided into three statuses: 500 Normal cases, 500 Pneumonia cases, and 125 COVID-19 positive cases. In 19], a screening model based on the transfer learning technique and the VGG19 network was reached an ACC rate of $93.48 \%$. The X-ray images they collected were split into three sets, one set of 504 Normal cases, one set of 700 Pneumonia cases, and one set of 224 COVID-19 patients (1427 cases in total). The authors of 37] obtained 96.9\% SEN, 97.5\% SPE, and 95\% ACC, which represents less than 1.43\%, 1.18 $\%$, and $1.91 \%$ of our results, respectively. CapsNet [43] reached $84.22 \%$ ACC for multi-class classification purposes. Table 4 shows the superiority of our method compared to the aforementioned methods. As expected, our method, which combines several components (i.e. a 2D CNN network, a building block of $3 \mathrm{D}$ convolutions, etc), outperforms other ones by achieving 98.33\% SEN, 98.68\% SPE, and $96.91 \%$ ACC, respectively. As seen, the proposed model exceeds the best competing model by an ACC rate of $1.91 \%$.

\subsection{Visualization}

In the supervised learning mode, end-to-end learning of a deep CNN is regarded as a black box. To date, visualizing and interpreting how CNNs interact has been a vital challenge for the computer vision community [40]. 


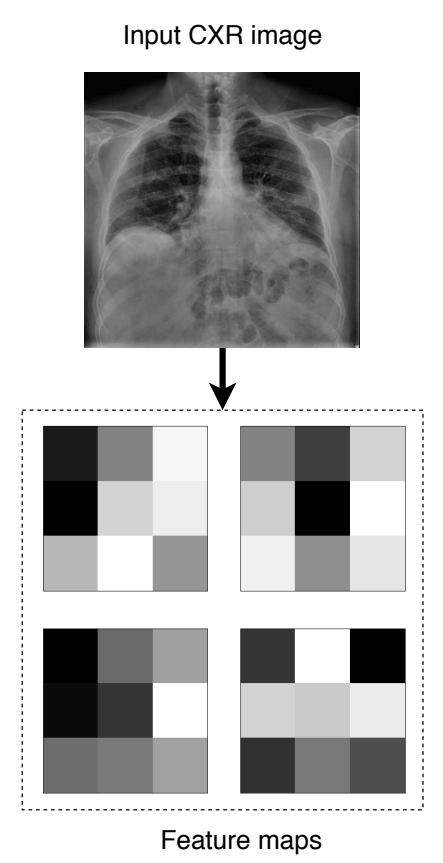

Fig. 10 Visualization of some of the feature maps produced by the output of the building block (3D CNN)

Fig. 10 shows how Hybrid-COVID trained by visualizing some of the feature maps generated by the 3D CNN output (building block). As can be seen, the representations learned are complex, rich, and informative. In general, the extracted features have become increasingly sophisticated at several levels of abstraction and representation 27. Therefore, the patterns learned with our Hybrid-COVID model pay more attention to contextual cues as well as the spatial aspect of the input image.

Besides, identifying regions where the disease is present is a vital task for further screening. The Grad-CAM [42] is the tool to predict the classdiscriminative localization map of an affected region in a seemingly unreasonable way. As shown in Fig. 11, the proposed Hybrid-COVID model gives a more accurate visual localization of the disease region.

\subsection{Impact of the hybridization pipeline}

To examine the impact of the hybridization pipeline on classification performance, we conducted another set of experiments under the following two scenarios:

- Scenario 1: Increase the number of 3D convolutions by a factor of 2 (i.e., make the 3D building block deeper by stacking a set of three 3D convolution layers with kernel sizes of $(3 \times 3 \times 3),(2 \times 2 \times 2)$ and $(1 \times 1 \times 1)$ respectively.); 
(a)
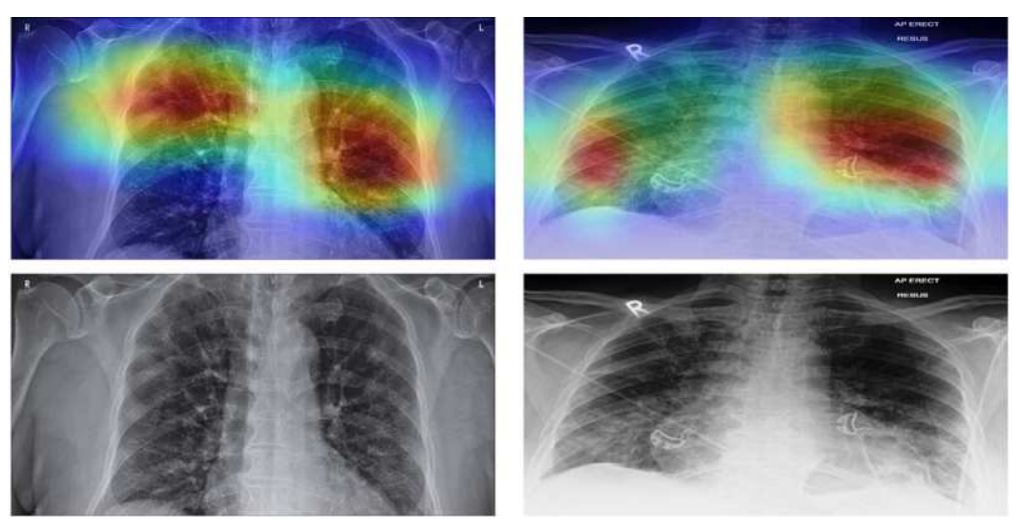

Fig. 11 CXR instances and corresponding localization maps: localization maps (a), CXR instances (b)

Table 5 Comparison of performance assessment between the baseline and two scenarios

\begin{tabular}{llll}
\hline Method & Scenario & Total parameters & ACC $(\%)$ \\
\hline Hybrid-COVID (2D CNN + 3D CNN) & Baseline & $17 \mathrm{M}$ & 96.91 \\
Hybrid-COVID (2D CNN + 3D CNN++) & 1 & $\approx 17 \mathrm{M}$ & 96.06 \\
Hybrid-COVID (2D CNN + 2D CNN) & 2 & $14 \mathrm{M}$ & 94.78 \\
\hline
\end{tabular}

- Scenario 2: Replace 3D convolutions with 2D counterparts (i.e., 2D CNN). Here, the reshaping operations are not necessary for space mapping. Specifically, the $2 \mathrm{D}$ convolution layers have kernel sizes of $(3 \times 3),(2 \times 2)$ and $(1 \times 1)$, respectively.

It should be noted that the hyper-parameter tuning and data distribution are the same as those presented in sub-section 2.3 and sub-section 2.1. respectively.

In what follows, we will examine the classification performance obtained under all scenarios. Table 5 displays the results obtained from the baseline scenario and the two other ones. Firstly, despite the increase in the depth of the network, we point out that the total number of parameters (i.e., trainable and non-trainable parameters) remains approximately the same. That is one of the reasons why transfer learning techniques make sense when combining $2 \mathrm{D}$ and 3D CNNs. As expected, the total number of parameters has decreased from $17 \mathrm{M}$ to $14 \mathrm{M}$, as a result of replacing $3 \mathrm{D}$ convolutions with $2 \mathrm{D}$ counterparts. Compared to 2D CNN, the number of parameters required for each 3D convolutional kernel is comparatively high. The overall ACC of the classification of the different scenarios is also given in Table 5. which shows that the baseline scenario performs better than the other two. Consequently, the two scenarios yield different results. However, the baseline scenario gives a good outcome with an ACC rate of $96.9 \%$, which is higher than the second (a difference of $0.85 \%$ ) and third (a difference of $2.13 \%$ ) scenarios. 


\section{Discussion}

The proposed transfer learning-based framework consists of a hybrid $2 \mathrm{D} / 3 \mathrm{D}$ CNN architecture that allows the automatic detection of COVID-19 using CXR modality. The implemented Hybrid-COVID model classifies each sample in a multi-class manner by predicting a class label for each testing example according to whether it belongs to the COVID-19 class, Normal, or Pneumonia. In this paper, Hybrid-COVID exploits the potential synergy between the pretrained VGG16 model (i.e., a 2D CNN) and a shallow 3D CNN for high-level contextual feature learning for each data sample. The proposed hybridization pipeline shows its proficiency in capturing both visual appearance and semantic detail from multi-dimensional feature space. The pipeline mainly consists of two correlated branches: the 2D CNN branch and the 3D CNN branch. On the one hand, the 2D CNN branch encodes features along the spatial axes of the data based on the transfer learning paradigm. On the other hand, the 3D CNN branch takes advantage of pre-trained parameters to capture and extract any inherent contextual information within the boundaries of SEN and SPE. In practice, the high SEN and SPE rates of a screening test are crucial for many reasons. For instance, a false negative result can lead to a critical situation where a patient with COVID-19 will not be isolated based on this test. However, this can lead to the widespread transmission of the virus to other healthy people, both indoors and outdoors. Compared to recent work in the literature, the experimental results produced by the Hybrid-COVID model show promising findings in terms of SPE and SEN. Besides, from Table 5 , we can see that the integration of a pre-trained deep 2D CNN with a shallow 3D CNN is the most efficient and accurate scenario to obtain better outcomes. Moreover, this research went beyond the limited availability of training data, as further modeling generally requires much more samples to achieve satisfactory results. Nevertheless, despite these results, the proposed Hybrid-COVID network still needs to be improved to meet the performance improvements expected by radiologists and health experts.

\section{Conclusion}

In this paper, a transfer learning-based hybrid 2D/3D CNN architecture has been developed to automatically detect and classify COVID-19 from CXR data. To do that, we performed an in-depth analysis to find a compromise between accuracy and efficiency by integrating both $2 \mathrm{D}$ and $3 \mathrm{D}$ CNNs into a hybrid design, called Hybrid-COVID. The findings of the proposed method clearly show its robustness compared to recent studies by up to $96.91 \%$ accuracy. We also showed that the integration of a depth-wise separable convolution and a spatial pyramid pooling module could produce high-level representations from multi-scale X-ray information. In the clinical practice, this high level of performance will help radiologists in making more informed and faster decisions to detect COVID-19 in the early stages. Finally, we consider that the 
proposed architecture can be further enhanced with larger datasets in the future.

\section{Compliance with ethical standards}

Funding This study did not receive external funding.

Conflict of interest The authors declare that they have no confict of interest.

\section{References}

1. World health organization (2020) Coronavirus. https://www.who.int/healthtopics/coronavirus. Accessed April 25, 2020

2. Bai Y, Yao L, Wei T, et al. (2020) Presumed Asymptomatic Carrier Transmission of COVID-19. JAMA 323(14):1406-1407. https://doi.org/10.1001/jama.2020.2565

3. Li R, et al. (2020) Substantial undocumented infection facilitates the rapid dissemination of novel coronavirus (SARS-CoV2). Science eabb3221. https://doi.org/10.1126/science.abb3221

4. Ai T, et al. (2020) Correlation of Chest CT and RT-PCR Testing in Coronavirus Disease 2019 (COVID-19) in China: A Report of 1014 Cases. Radiology 200642. https://doi.org/10.1148/radiol.2020200642

5. Dangis A, et al. (2020) Accuracy and reproducibility of low-dose submillisievert chest CT for the diagnosis of COVID-19. Radiol Cardiothorac Imaging 2(2):e200196. https://doi.org/10.1148/ryct.2020200196

6. Fang Y, et al. (2020) Sensitivity of Chest CT for COVID-19: Comparison to RT-PCR Radiology 200432. https://doi.org/10.1148/radiol.2020200432

7. Bai HX, et al. (2020) Performance of radiologists in differentiating COVID-19 from vira pneumonia on chest CT. Radiology 200823 https://doi.org/10.1148/radiol.2020200823

8. Zhao D, et al. (2020) A comparative study on the clinical features of COVID-19 pneumonia to other pneumonias. Clin Infect Dis ciaa247 https://doi.org/10.1093/cid/ciaa247.

9. Jin S, et al. (2020) AI-assisted CT imaging analysis for COVID-19 screening: Building and deploying a medical AI system in four weeks. medRxiv 2020.03.19.20039354. https://doi.org/10.1101/2020.03.19.20039354

10. Butt C, Gill J, Chun D, et al. (2020) Deep learning system to screen coronavirus disease 2019 pneumonia. Appl Intell. https://doi.org/10.1007/s10489-020-01714-3

11. Willemink MJ, Noël PB. (2019) The evolution of image reconstruction for CT-from filtered back projection to artificial intelligence. Eur Radiol 29(5):2185-2195. https://doi.org/10.1007/s00330-018-5810-7

12. Jacobi A, Chung M, Bernheim A, et al. (2020) Portable chest X-ray in coronavirus disease-19 (COVID-19): A pictorial review. Clin Imaging 64:35-42. https://doi.org/10.1016/j.clinimag.2020.04.001

13. Cohen JP, Morrison P, Dao L. (2020) COVID-19 Image Data Collection. arXiv [csCV]

14. Chest X-Ray Images (Pneumonia) (2020) https://www.kaggle.com/ paultimothymooney/chest-xray-pneumonia. Accessed June 25, 2020

15. Simonyan K, Zisserman A (2015) Very deep convolutional networks for large-scale image recognition. arXiv:1409.1556

16. Chollet F. Xception (2017) Deep Learning with Depthwise Separable Convolutions. In: CVPR, pp. 1800-1807. https://doi.org/10.1109/CVPR.2017.195

17. He K, et al. (2014) Spatial Pyramid Pooling in Deep Convolutional Networks for Visual Recognition. In: ECCV, pp. 346-361

18. Narin A, Kaya C, Pamuk Z (2020) Automatic Detection of Coronavirus Disease (COVID-19) Using X-ray Images and Deep Convolutional Neural Networks. arXiv: 2003.10849 
19. Apostolopoulos ID, Mpesiana TA (2020) Covid-19: automatic detection from X-ray images utilizing transfer learning with convolutional neural networks. Phys Eng Sci Med $1-6$

20. Karim MR,Döhmen T, Rebholz-Schuhmann D, et al. (2020) DeepCOVIDExplainer: Explainable COVID-19 Predictions Based on Chest X-ray Images. arXiv: 2004.04582

21. Basu S, Mitra S, Saha N (2020) Deep Learning for Screening COVID-19 using Chest X-Ray Images. arXiv:2004.10507

22. Hemdan EE-D, Shouman MA, Karar ME (2020) COVIDX-Net: A Framework of Deep Learning Classifiers to Diagnose COVID-19 in X-Ray Images. arXiv:2003.11055

23. He K, et al. (2015) Deep Residual Learning for Image Recognition. arXiv:1512.03385

24. Fan L, et al. (2020) Progress and prospect on imaging diagnosis of COVID-19. Chin J Acad Radiol 3(1):4-13. https://doi.org/10.1007/s42058-020-00031-5

25. Chaari L, Golubnitschaja O (2020) Covid-19 pandemic by the 'real-time' monitoring: the Tunisian case and lessons for global epidemics in the context of 3PM strategies. EPMA J . https://doi.org/10.1007/s13167-020-00207-0

26. Khan A, et al. (2020) A Survey of the Recent Architectures of Deep Convolutional Neural Networks. arXiv:1901.06032

27. LeCun Y, et al. (2015) Deep learning. Nature 521(7553):436-444. https://doi.org/10.1038/nature14539azz.

28. Kingma DP, Ba J (2014) Adam: A Method for Stochastic Optimization. arXiv:1412.6980

29. Srivastava N, et al. (2014) Dropout: A Simple Way to Prevent Neural Networks from Overfitting. J Mach Learn Res 15(56):1929-1958

30. Shorten C, Khoshgoftaar TM (2019) A survey on Image Data Augmentation for Deep Learning. J Big Data 6(1):60. https://doi.org/10.1186/s40537-019-0197-0

31. Ng M-Y, et al. (2020) Imaging Profile of the COVID-19 Infection: Radiologic Findings and Literature Review. Radiol Cardiothorac Imaging 2(1): e200034. https://doi.org/10.1148/ryct.2020200034

32. Bayoudh K, et al. (2020) Transfer learning based hybrid 2D-3D CNN for traffic sign recognition and semantic road detection applied in advanced driver assistance systems. Appl Intell. https://doi.org/10.1007/s10489-020-01801-5

33. Zhang L. (2019) Transfer Adaptation Learning: A Decade Survey. arXiv:1903.04687

34. Ji, S., Xu, W., Yang, M., Yu, K (2013) 3D Convolutional Neural Networks for Human Action Recognition. IEEE Transactions on Pattern Analysis and Machine Intelligence 35, 221-231

35. COVID-19 Radiography Database (2020) https://www.kaggle.com/tawsifurrahman/covid19radiography-database. Accessed June 25, 2020

36. Ozturk, T. et al. (2020) Automated detection of COVID-19 cases using deep neural networks with X-ray images. Computers in Biology and Medicine 121:103792

37. Toğaçar, M., Ergen, B., Cömert, Z (2020) COVID-19 detection using deep learning models to exploit Social Mimic Optimization and structured chest X-ray images using fuzzy color and stacking approaches. Computers in Biology and Medicine 121:103805

38. Long, J., Shelhamer, E., Darrell, T. Fully (2015) Convolutional Networks for Semantic Segmentation. arXiv:1411.4038

39. Tran, D., Bourdev, L., Fergus, R., Torresani, L., Paluri, M. (2015) Learning Spatiotemporal Features with 3D Convolutional Networks. arXiv:1412.0767

40. Zeiler, M. D., Fergus, R (2013) Visualizing and Understanding Convolutional Networks. arXiv:1311.2901

41. Redmon, J., Divvala, S., Girshick, R., Farhadi, A (2016) You Only Look Once: Unified, Real-Time Object Detection. arXiv:1506.02640

42. Selvaraju, R. R. et al. (2020) Grad-CAM: Visual Explanations from Deep Networks via Gradient-based Localization. Int J Comput Vis 128:336-359

43. Toraman, S., Alakus, T. B., Turkoglu, I (2020) Convolutional capsnet: A novel artificial neural network approach to detect COVID-19 disease from X-ray images using capsule networks. Chaos, Solitons \& Fractals 140:110122

44. Kwabena Patrick, M., Felix Adekoya, A., Abra Mighty, A., Edward, B. Y (2019) Capsule Networks - A survey. Journal of King Saud University - Computer and Information Sciences. doi:10.1016/j.jksuci.2019.09.014 
45. Khan, A. I., Shah, J. L., Bhat, M. M (2020) CoroNet: A deep neural network for detection and diagnosis of COVID-19 from chest x-ray images. Computer Methods and Programs in Biomedicine 196:105581

46. Das, D., Santosh, K. C., Pal, U (2020) Truncated inception net: COVID-19 outbreak screening using chest X-rays. Phys Eng Sci Med. doi:10.1007/s13246-020-00888-x

47. Brady, Z. et al (2020) Technique, radiation safety and image quality for chest X-ray imaging through glass and in mobile settings during the COVID-19 pandemic. Phys Eng Sci Med. doi:10.1007/s13246-020-00899-8 
Figures

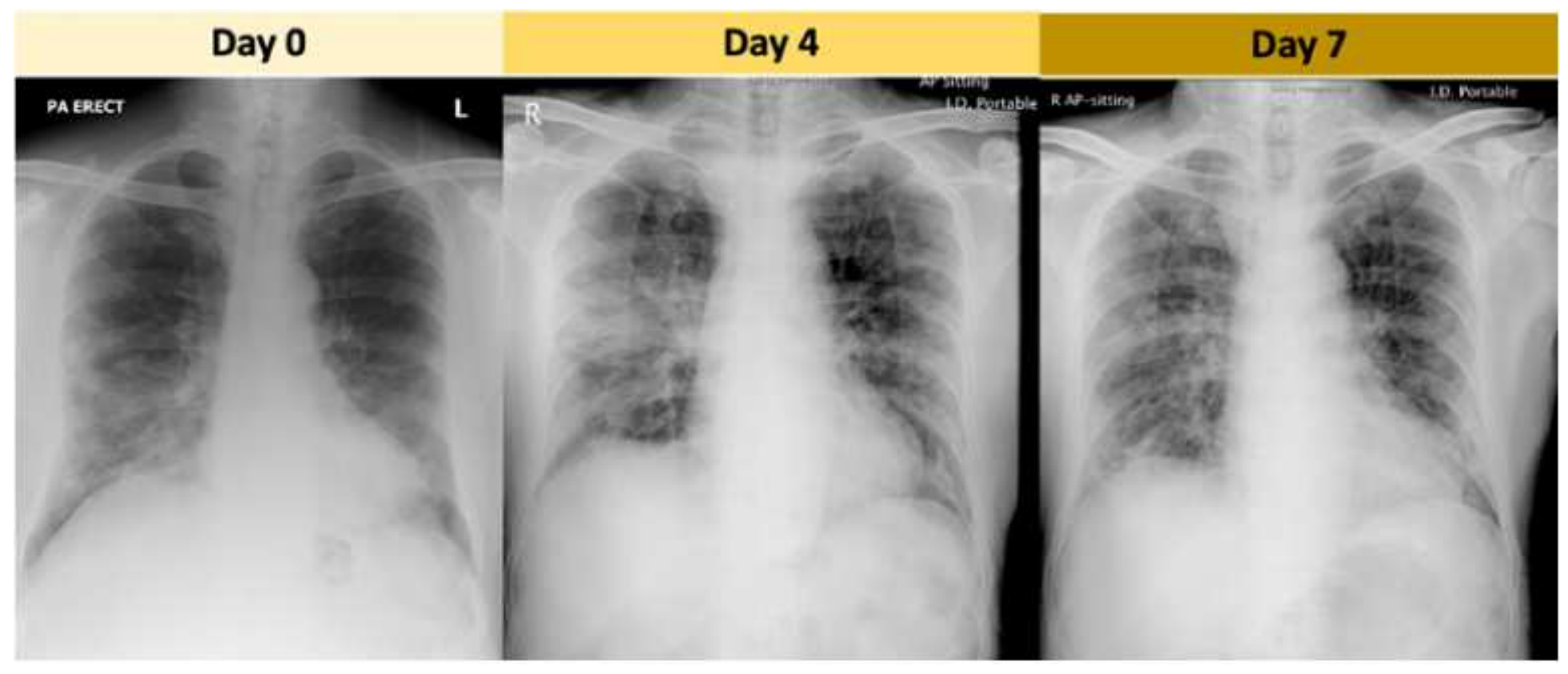

Figure 1

Temporal evolution of CXR outcomes in an older male patient with 3 consolidation changes in the lung periphery

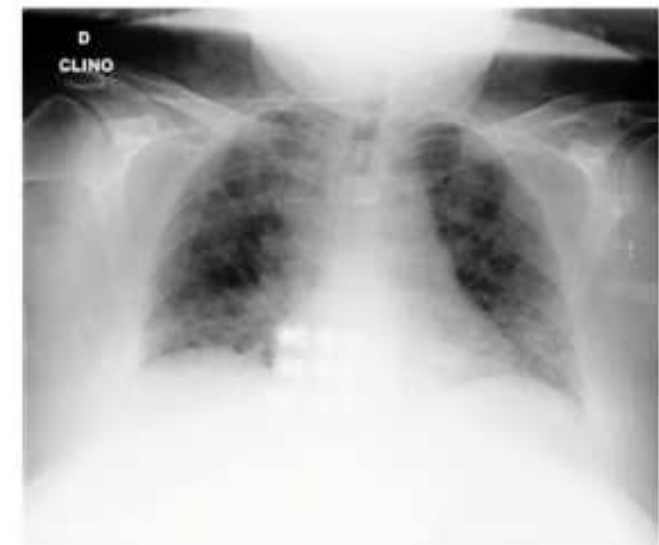

(a)

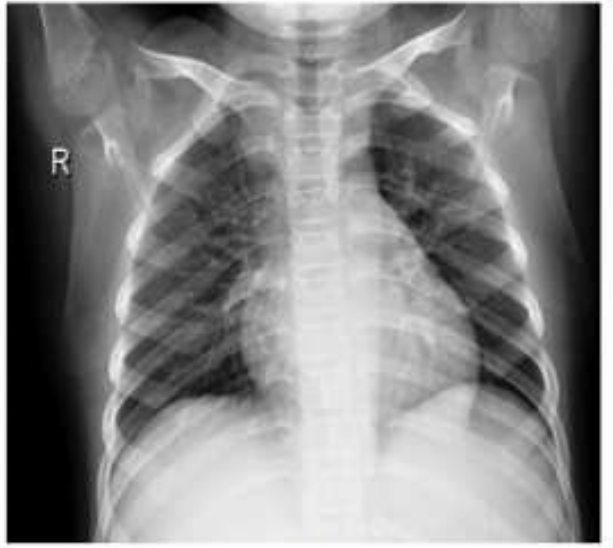

(b)

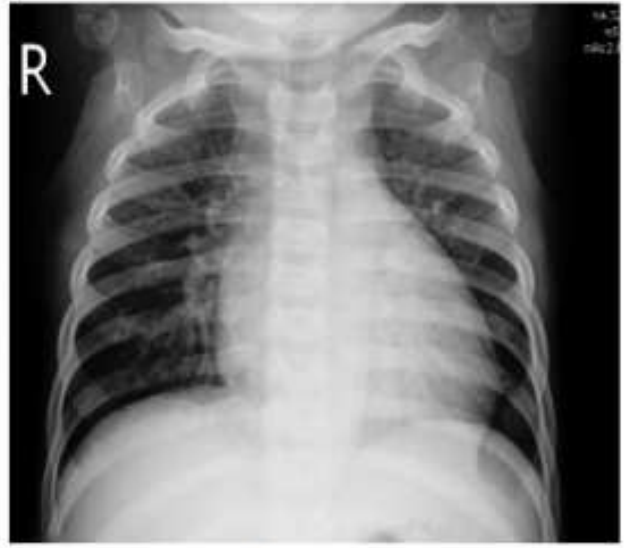

(c)

Figure 2

A selection of CXR samples taken from the collected dataset: a Normal case (a), a COVID-19 patient (b), a Pneumonia case (c) 
The collected dataset

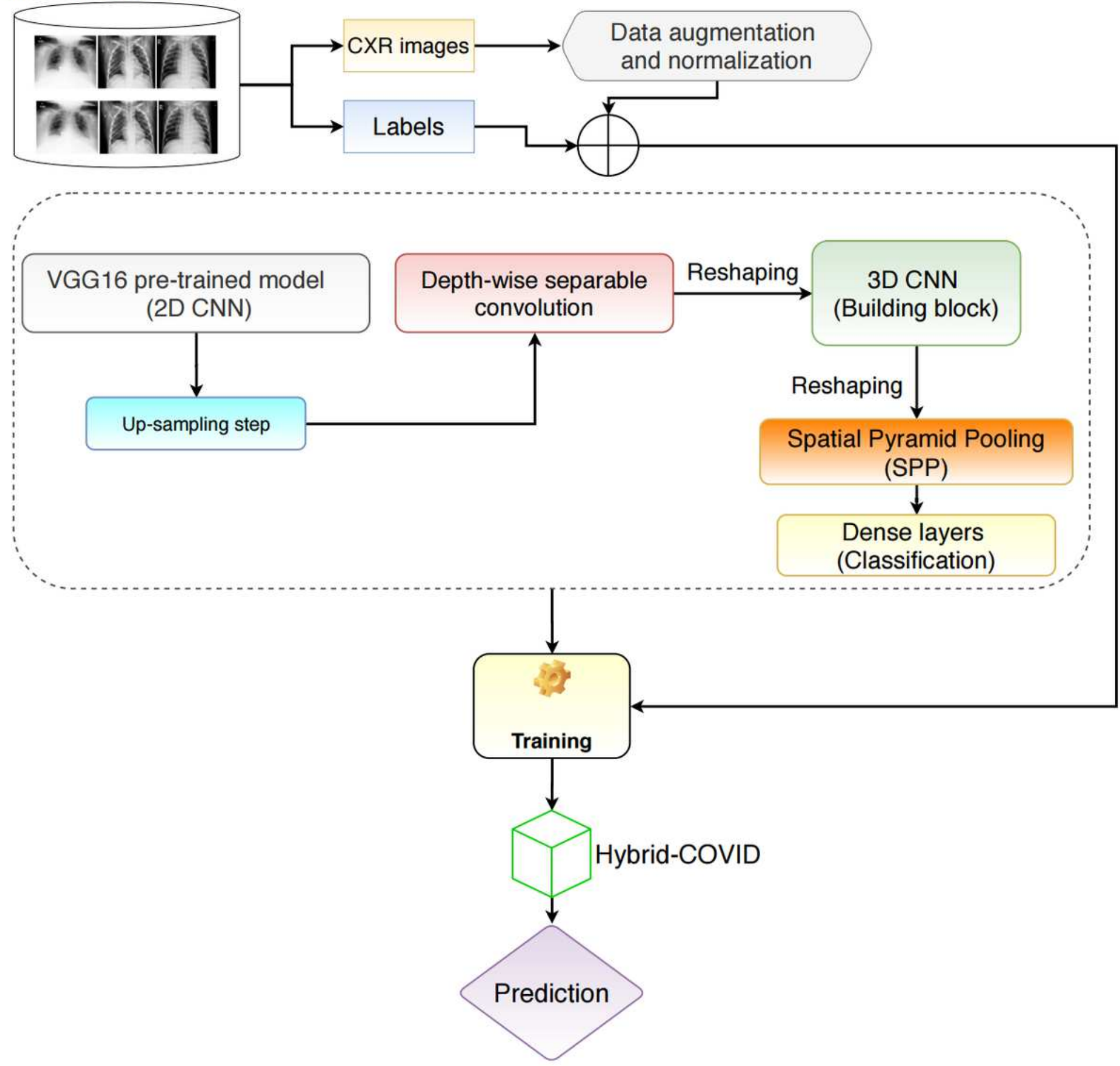

Figure 3

Workflow diagram of the proposed framework 


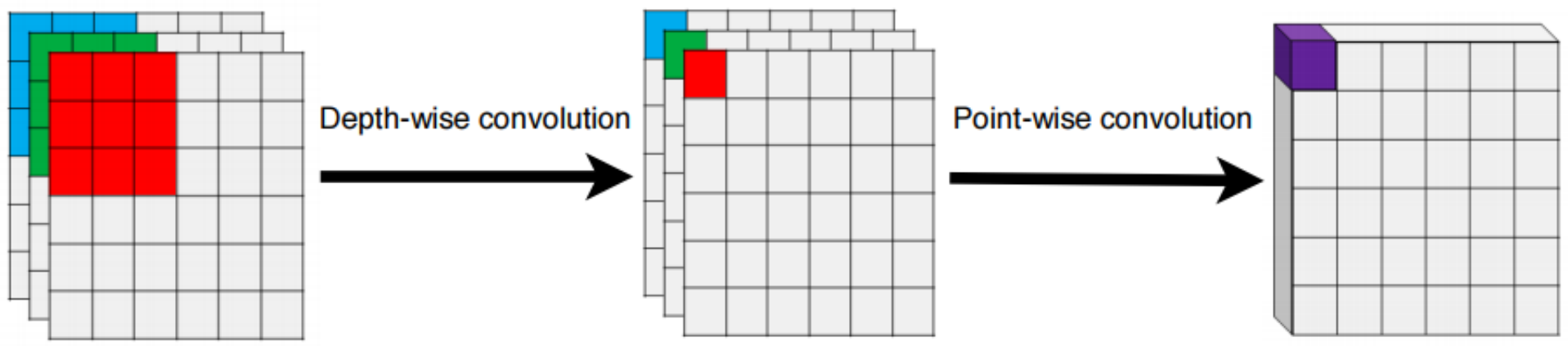

Figure 4

Depth-wise separable convolution steps
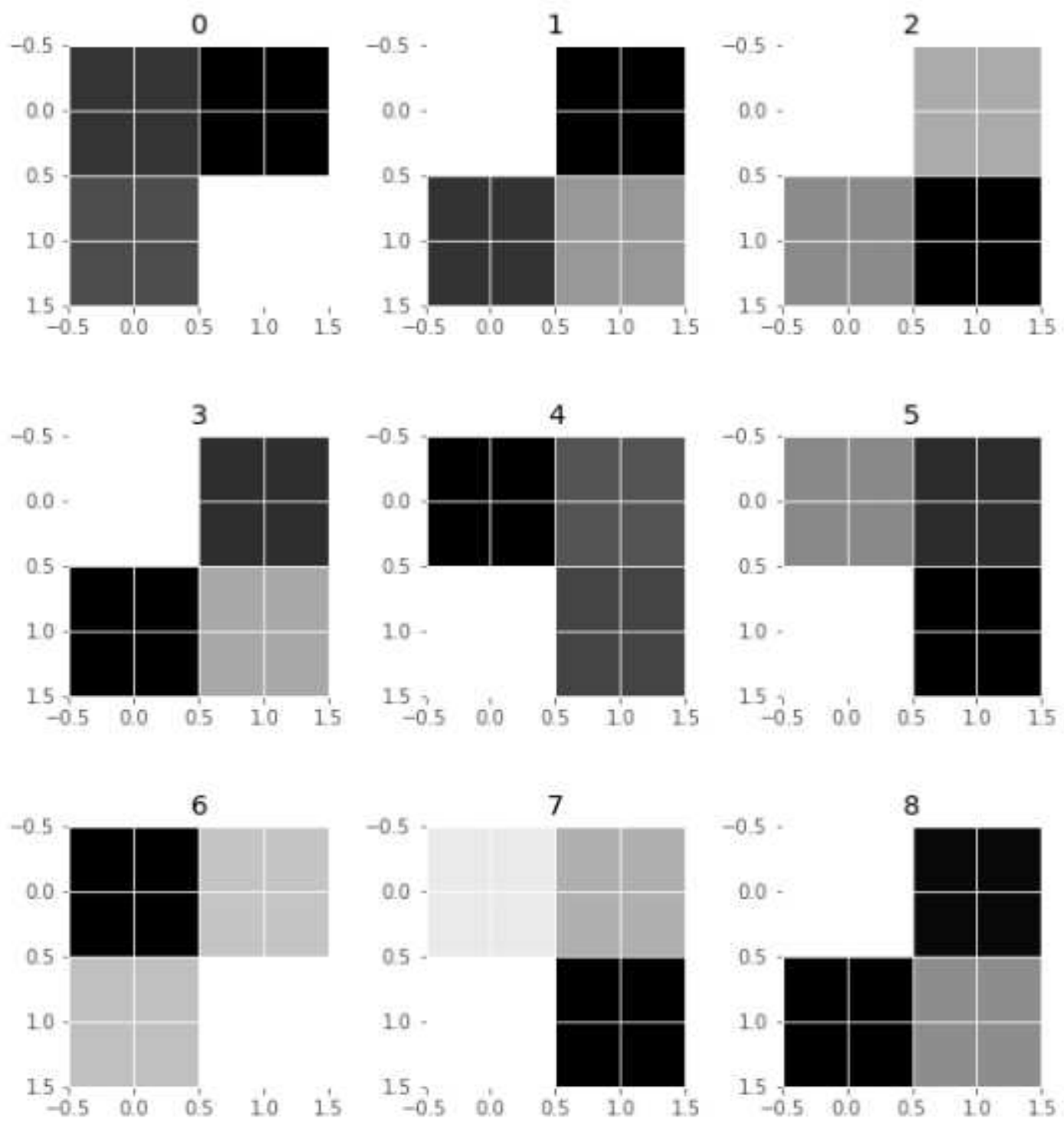

Figure 5

Visualization of some learned weights from the depth-wise separable convolution layer 


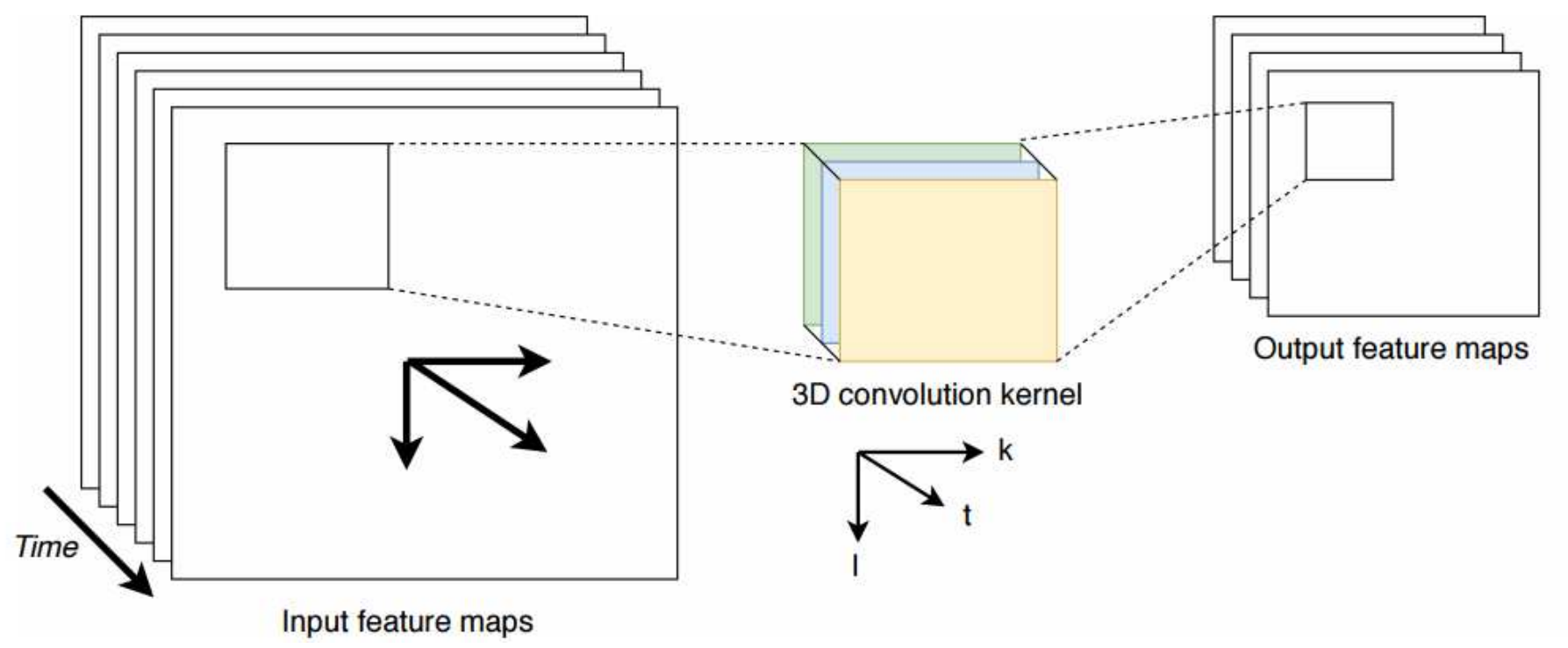

Figure 6

A schematic illustration of a 3D convolution layer

Fixed-length representation

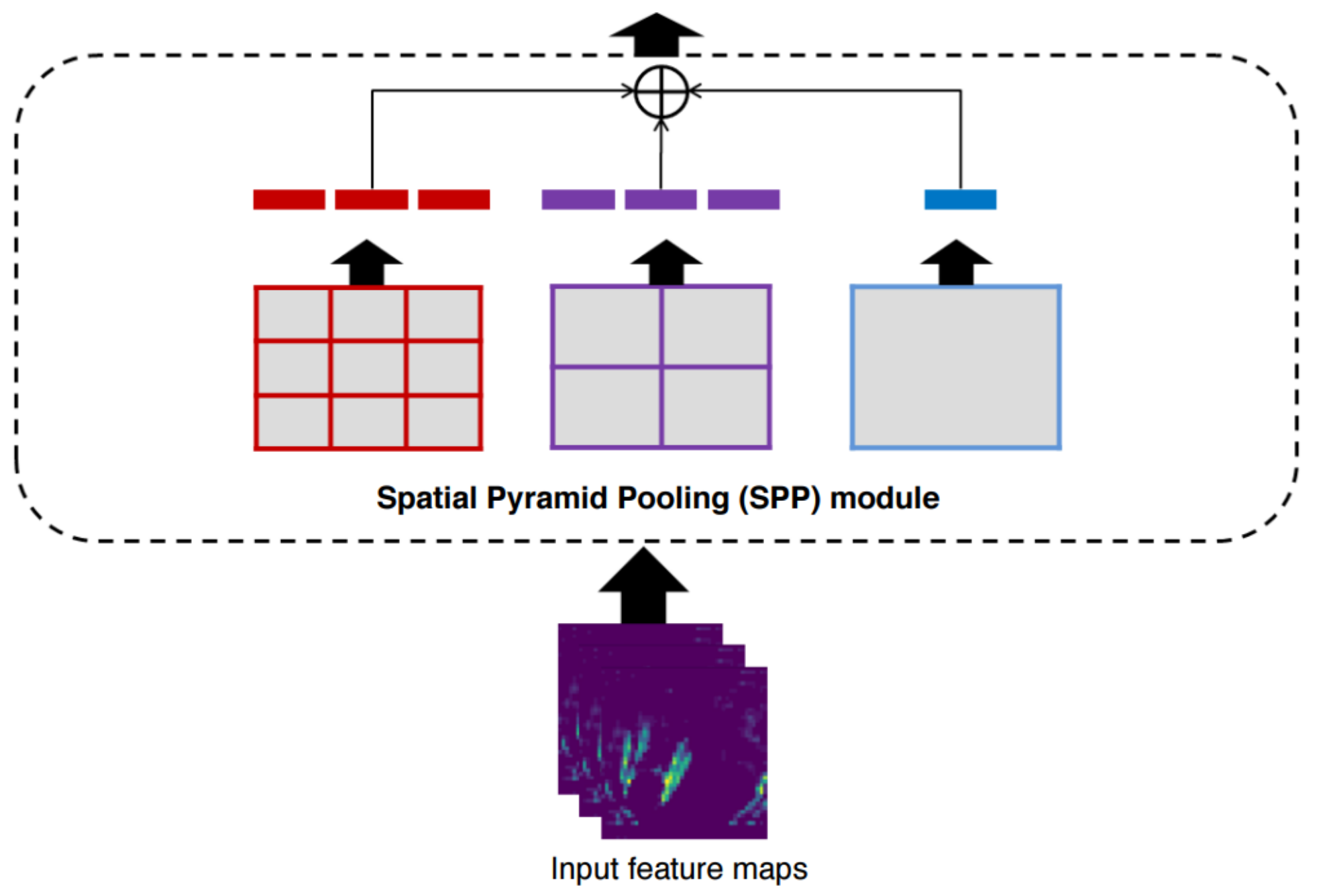

Figure 7 
Proposed spatial pyramid pooling (SPP) structure. Here, 3 level pyramid pooling is applied: $3 \times 3,2 \times 2$, and $1 \times 1$, respectively

\section{Training and validation loss and accuracy results}

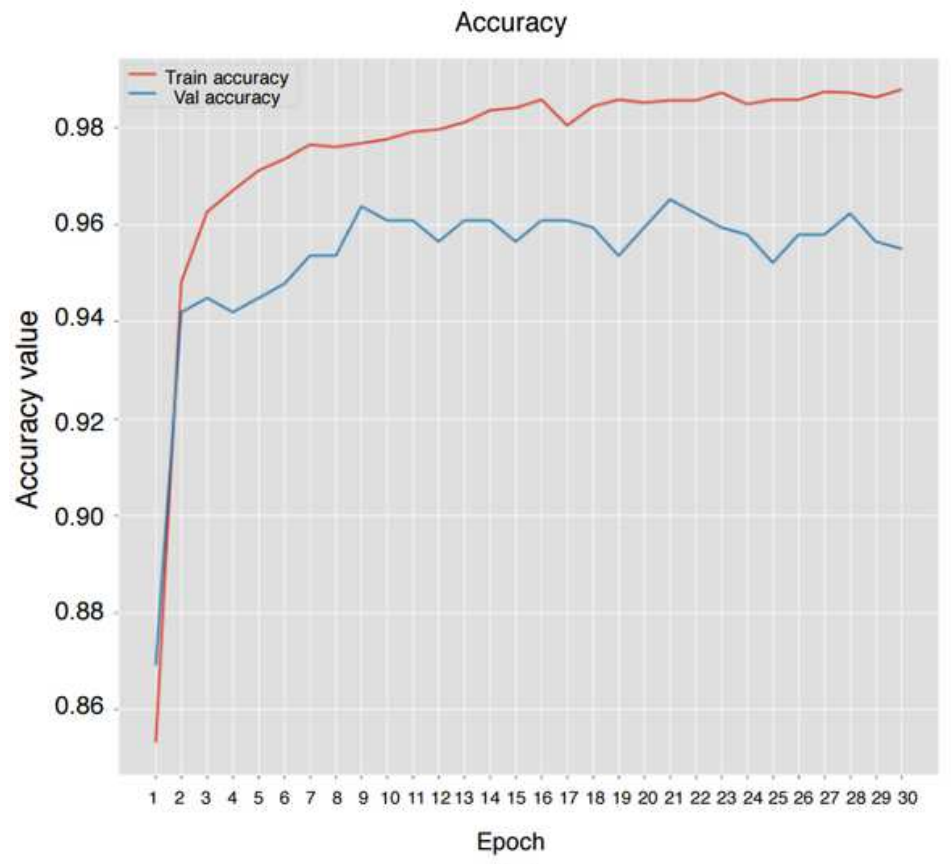

(a)

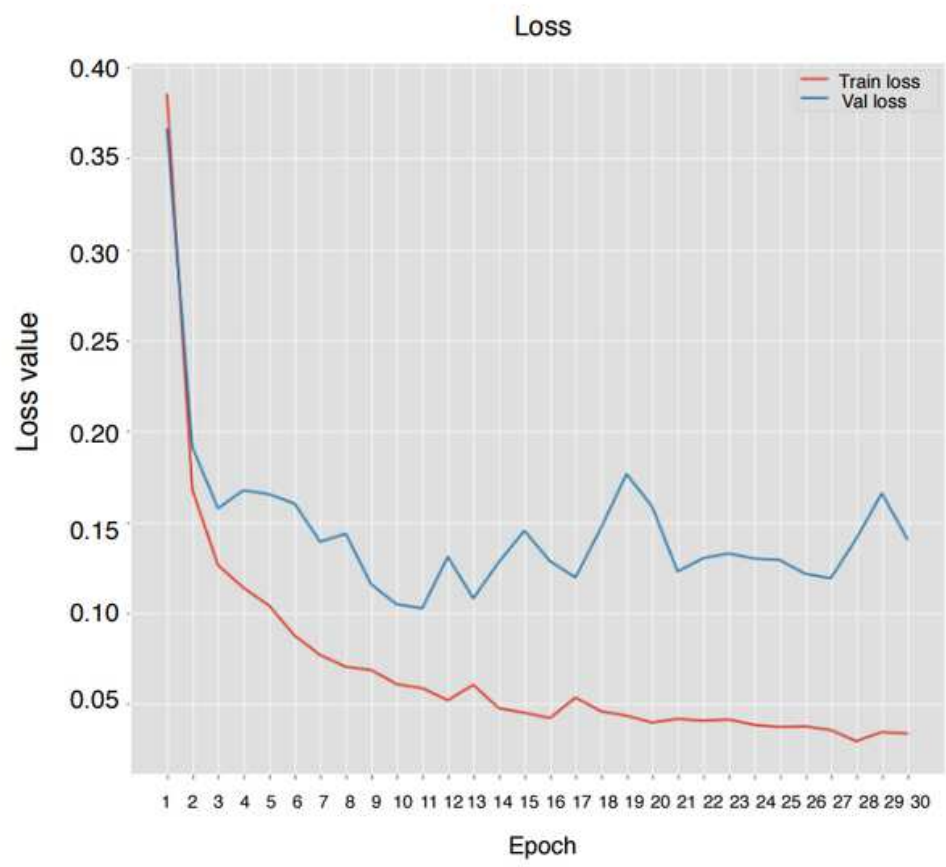

(b)

\section{Figure 8}

Visualization of model performance per epoch for: training and validation accuracy (a), training and validation loss (b) 


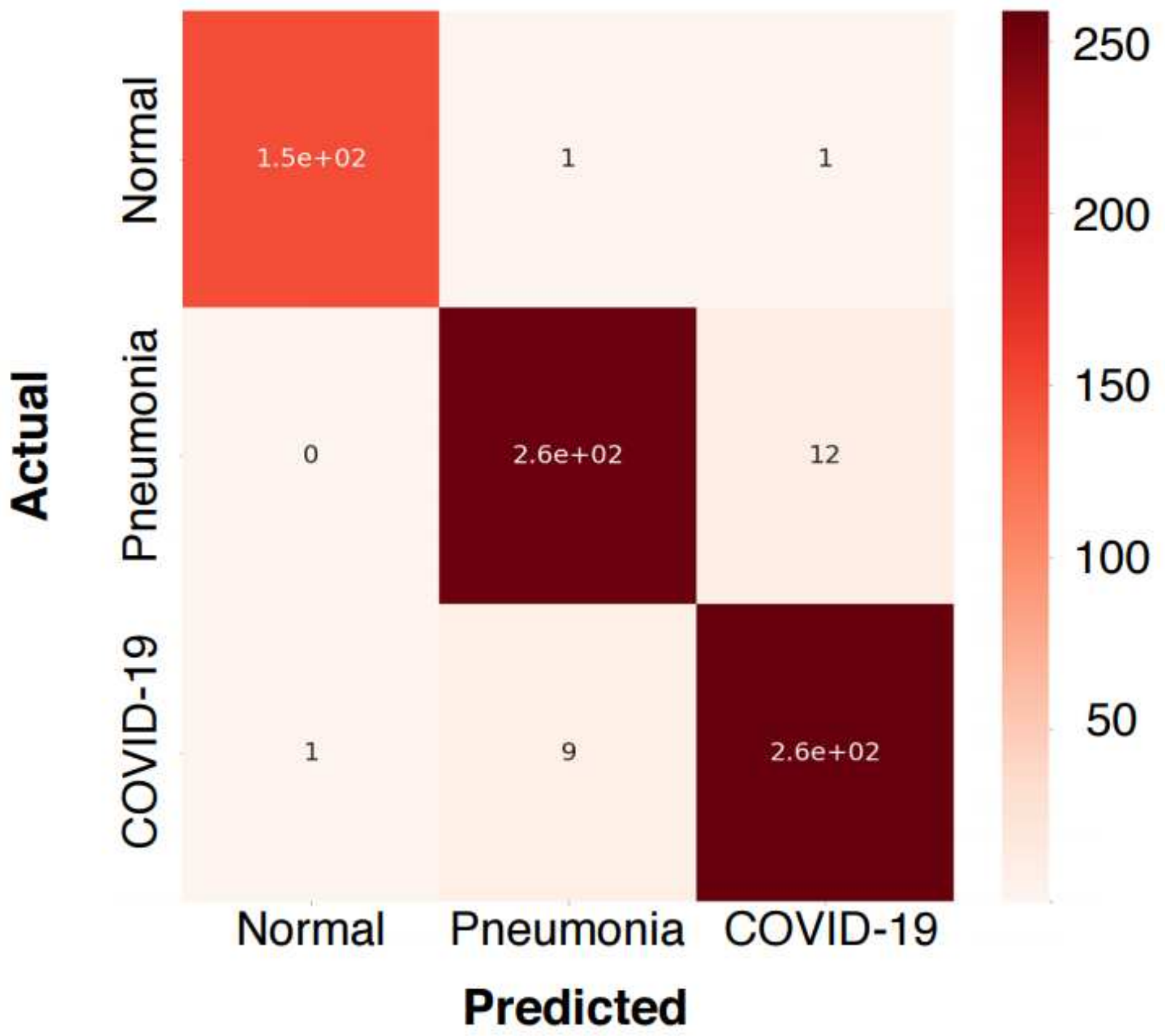

Figure 9

Confusion matrix of the Hybrid-COVID model 


\section{Input CXR image}

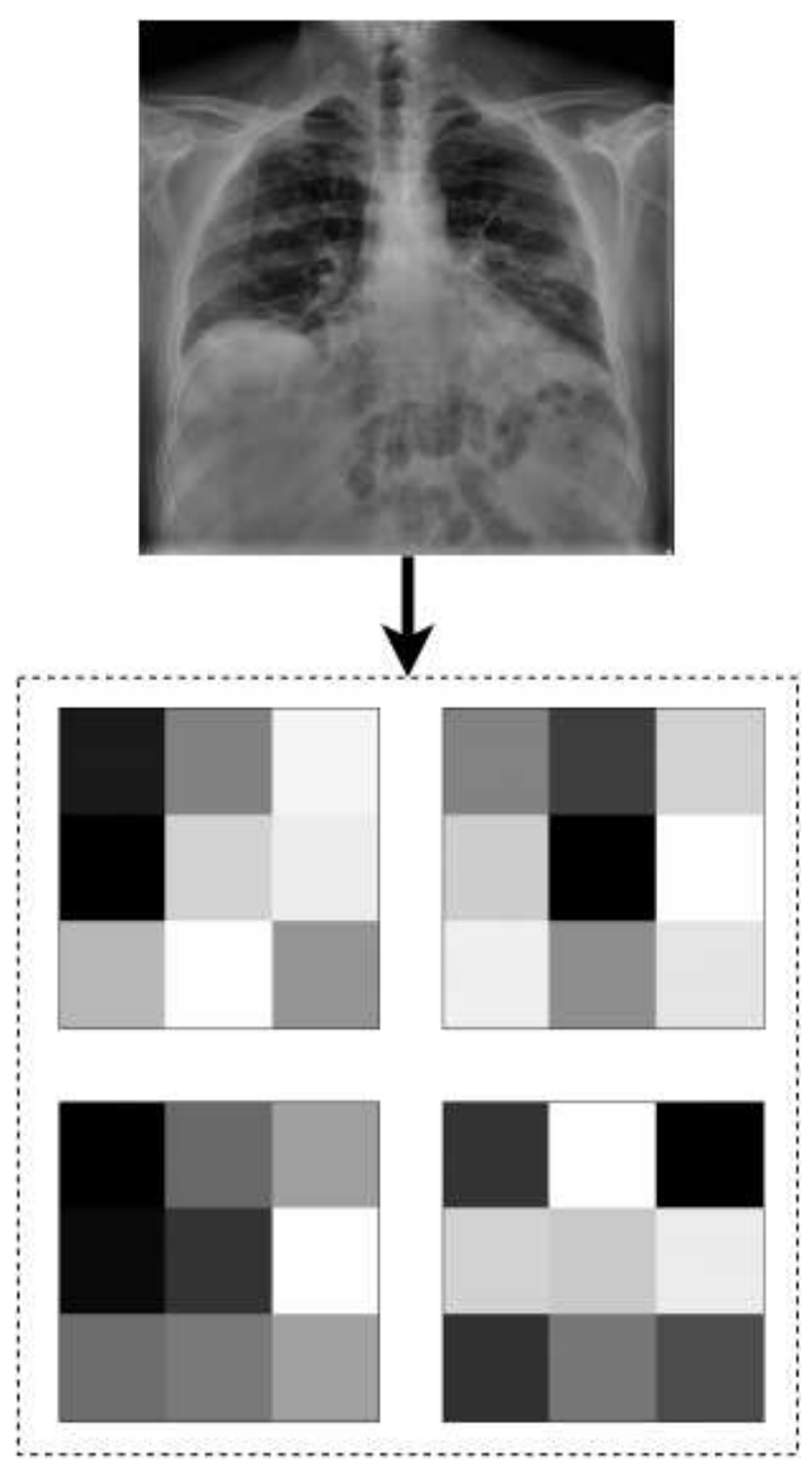

\section{Feature maps}

Figure 10

Visualization of some of the feature maps produced by the output of the building block (3D CNN) 
(a)
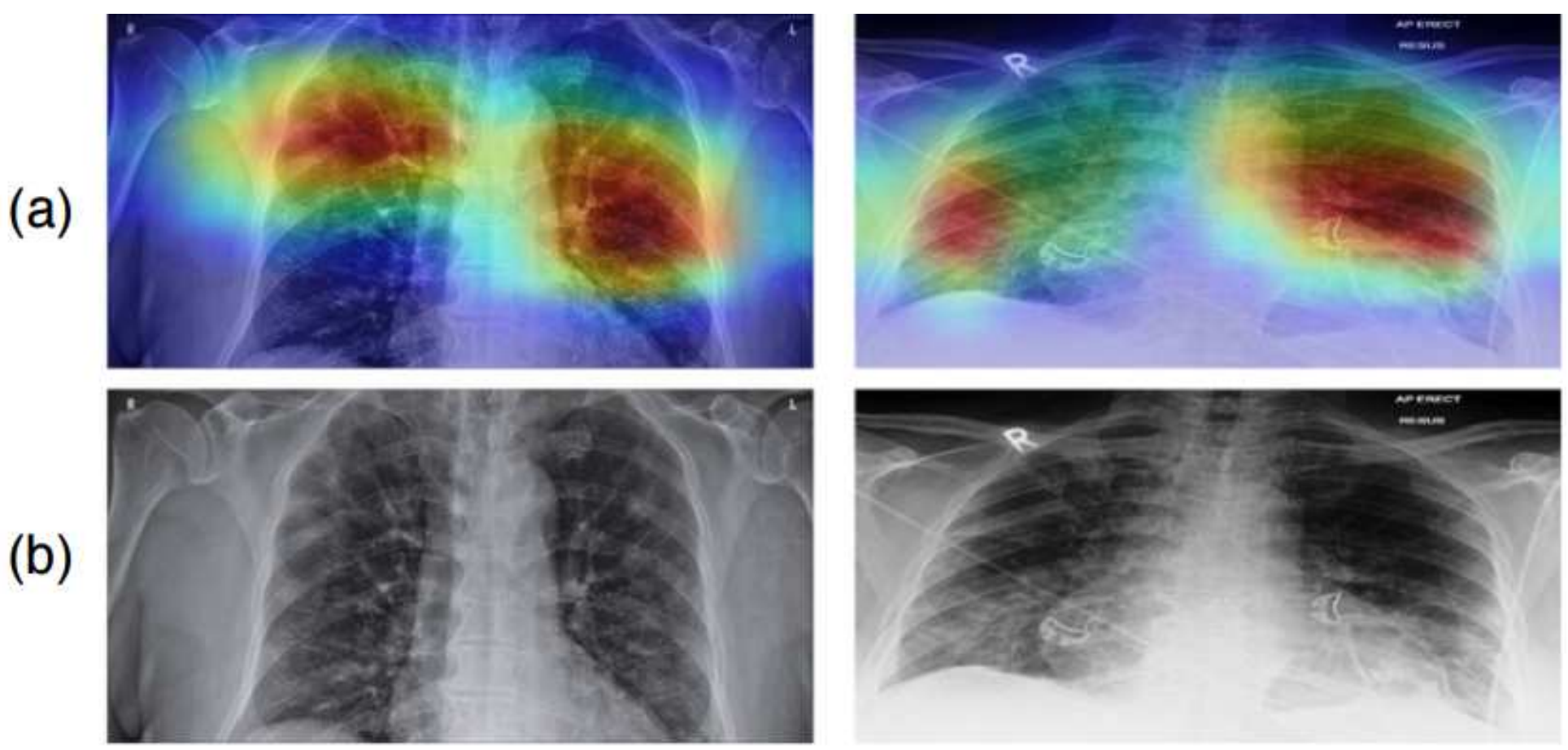

Figure 11

CXR instances and corresponding localization maps: localization maps (a), CXR instances (b) 